\title{
CANONICAL PARAMETERIZATIONS OF METRIC DISKS
}

\author{
ALEXANDER LYTCHAK and STEFAN WENGER
}

\begin{abstract}
We use the recently established existence and regularity of area and energy minimizing disks in metric spaces to obtain canonical parameterizations of metric surfaces. Our approach yields a new and conceptually simple proof of a well-known theorem of Bonk and Kleiner on the existence of quasisymmetric parameterizations of linearly locally connected, Ahlfors 2-regular metric 2-spheres. Generalizations and applications to the geometry of such surfaces are described.
\end{abstract}

\section{Introduction and statement of main results}

By the classical uniformization theorem, every smooth Riemann surface is conformally diffeomorphic to a surface of constant curvature. A fundamental question, widely studied in the field of analysis in metric spaces, asks to what extent nonsmooth metric spaces admit parameterizations from a Euclidean domain with good geometric and analytic properties. In this more general context, one usually looks for parameterizations which are bi-Lipschitz, quasisymmetric, or quasiconformal.

A celebrated and difficult theorem of Bonk-Kleiner [4] asserts that an Ahlfors 2-regular metric space $X$, homeomorphic to the standard 2-sphere $S^{2}$, admits a quasisymmetric homeomorphism from $S^{2}$ to $X$ if and only if $X$ is linearly locally connected. We refer to Section 2 for the definitions of quasisymmetric homeomorphism, linear local connectedness, and Ahlfors 2-regularity. This result has since been extended, for example, in [5], [34], [47], [48], and [26], and recently in the important paper [37]. We refer to [37] for details and more references.

The main purpose of the present paper is to provide a new and conceptually simple approach to the theorem of Bonk and Kleiner and related results. Our approach is a direct generalization of the classical existence proof of conformal parameterizations of smooth surfaces via minimization of the energy of maps into the surface (see [18]). In a similar vein, energy-minimizing maps into smooth surfaces and into 
surfaces with upper curvature bounds and conical singularities have been used in [19], [23], [35], [38], [40], and [36], where their injectivity, optimal quasiconformality, and connection with Teichmüller theory are studied. Our main parameterization result can be stated as follows. We denote by $D$ and $\bar{D}$ the open and closed unit disks in $\mathbb{R}^{2}$, respectively, and refer to Section 2 for the definition of energy $E_{+}^{2}(u)$ of a (Sobolev) map $u$ from $D$ to a metric space.

\section{THEOREM 1.1}

Let $X$ be a geodesic metric space homeomorphic to $\bar{D}$ and with boundary circle $\partial X$ of finite length. If $X$ is Ahlfors 2-regular and linearly locally connected, then there exists a homeomorphism $u: \bar{D} \rightarrow X$ of minimal energy $E_{+}^{2}(u)<\infty$. Any such $u$ is quasisymmetric and is uniquely determined up to a conformal diffeomorphism of $\bar{D}$.

A more general statement will be provided in Theorem 6.1. Note that the theorem comprises several statements, which will be described more precisely and in a different order below. First, there exists a continuous map of finite energy from $\bar{D}$ to $X$ whose boundary parameterizes the boundary circle $\partial X$. Second, there exists one such map of minimal energy. Finally, any such map is a quasisymmetric homeomorphism which is unique up to composition with a conformal diffeomorphism of $\bar{D}$. Similarly to Theorem 1.1, we obtain a canonical (up to conformal diffeomorphism) quasisymmetric parameterization of Ahlfors 2-regular, linearly locally connected metric 2-spheres (see Theorem 6.2) and, consequently, the Bonk-Kleiner theorem mentioned above (see Corollary 6.3).

We now describe our approach and the statements of the theorem in more precise terms. Let $X$ be a complete metric space. Denote by $N^{1,2}(D, X)$ the space of (Newton-) Sobolev maps from $D$ to $X$ in the sense of [17]. Given a map $u \in$ $N^{1,2}(D, X)$, we let $\operatorname{tr}(u)$ be its trace, $E_{+}^{2}(u)$ its Reshetnyak energy, and Area $(u)$ its parameterized area. See Section 3 for these definitions and references. If $\Gamma \subset X$ is a Jordan curve, then we let $\Lambda(\Gamma, X)$ be the possibly empty family of maps $u \in$ $N^{1,2}(D, X)$ whose trace has a continuous representative which is a weakly monotone parameterization of $\Gamma$.

Our first result provides topological information on energy minimizers.

\section{THEOREM 1.2}

Let $X$ be a geodesic metric space homeomorphic to $\bar{D}$, and let $u: \bar{D} \rightarrow X$ be a continuous map. If $u$ is in $\Lambda(\partial X, X)$ and minimizes the Reshetnyak energy $E_{+}^{2}$ among all maps in $\Lambda(\partial X, X)$, then $u$ is a uniform limit of homeomorphisms from $\bar{D}$ to $X$.

If $X$ has a quadratic bound $\mathscr{H}^{2}(B(x, r)) \leq C \cdot r^{2}$ for the Hausdorff 2-measure of $r$-balls, then any $u$ as in the theorem above is a homeomorphism (see Theorem 3.6). 
In general, the family $\Lambda(\partial X, X)$ may be empty for spaces as in Theorem 1.2. However, for spaces admitting a quadratic isoperimetric inequality as defined below, this family is not empty when the length of $\partial X$ is finite. Furthermore, energy minimizers exist, are continuous, and their infinitesimal structure is as close to conformal as possible by our results in [29] and [30] (see also Theorem 3.4).

\section{Definition 1.3}

A complete metric space $X$ is said to admit a quadratic isoperimetric inequality if there exists $C>0$ such that every Lipschitz curve $c: S^{1} \rightarrow X$ is the trace of some $u \in N^{1,2}(D, X)$ such that

$$
\operatorname{Area}(u) \leq C \cdot \ell(c)^{2},
$$

where $\ell(c)$ denotes the length of $c$.

If the constant $C$ matters, then we will say that $X$ admits a quadratic isoperimetric inequality with (isoperimetric) constant $C$. Our second ingredient in the proof of Theorem 1.1 is the following.

THEOREM 1.4

Let $X$ be a complete, geodesic metric space homeomorphic to $\bar{D}, S^{2}$, or $\mathbb{R}^{2}$. Suppose that there exists $C>0$ such that every Jordan curve in $X$ bounds a Jordan domain $U \subset X$ with

$$
\mathscr{H}^{2}(U) \leq C \cdot \ell(\partial U)^{2} .
$$

Then $X$ admits a quadratic isoperimetric inequality with constant $C$.

A Jordan domain $U \subset X$ is an open set homeomorphic to $D$ such that $\bar{U} \subset$ $X$ is homeomorphic to $\bar{D}$. Particular examples of spaces satisfying (1) are Ahlfors 2-regular, linearly locally connected metric spaces homeomorphic to $\bar{D}$ or $S^{2}$ (see Lemma 5.6).

Now, if $X$ is as in Theorem 1.1, then it admits a quadratic isoperimetric inequality by Theorem 1.4. Hence, the family $\Lambda(\partial X, X)$ is not empty and, by [29] and [30], contains an energy minimizer $u$ which is continuous. By Theorem 1.2 and the remark following it, any such $u$ is a homeomorphism. Since the infinitesimal structure of energy minimizers is as close to conformal as possible by [29] and [30], the modulus estimates from [15] imply that $u$ is quasisymmetric; furthermore, $u$ is uniquely determined up to a conformal diffeomorphism of $\bar{D}$. This concludes the outline of the proof of Theorem 1.1.

The class of metric surfaces satisfying (1) is of importance in the theory of minimal surfaces in metric spaces (see [31]). Such surfaces need not be Ahlfors 2-regular 
and they need not admit quasiconformal parameterizations (as defined in [37]) even locally (see Example 5.9). Combining Theorems 1.4 and 1.2, we nevertheless obtain natural "almost parameterizations" of such surfaces (see Theorem 5.8). For surfaces satisfying (1) with the Euclidean constant $C=\frac{1}{4 \pi}$, we obtain, as a consequence of Theorem 1.4 together with [32, Theorem 1.1], the following strengthening of [32, Theorem 1.3].

\section{COROLLARY 1.5}

Let $X$ be a geodesic metric space homeomorphic to $\bar{D}$. Then $X$ is a CAT(0)-space if and only if every Jordan domain $\Omega \subset X$ satisfies

$$
\mathscr{H}^{2}(\Omega) \leq \frac{1}{4 \pi} \cdot \ell(\partial \Omega)^{2} .
$$

In particular, spaces as in the corollary are Lipschitz 1-connected (see Section 5 below and [25]). For spaces satisfying (1) with $C>\frac{1}{4 \pi}$, it is in general not easy to construct nontrivial maps with suitable metric or analytic properties, as the following open problem shows.

\section{QUESTION 1.6}

Let $X$ be a geodesic, Ahlfors 2-regular, linearly locally connected metric space homeomorphic to $\bar{D}$. Is it true that every Lipschitz map $c: S^{1} \rightarrow X$ extends to a Lipschitz map on $\bar{D}$ ? If so, is X Lipschitz 1-connected?

Notice that Theorem 1.4 in particular asserts the existence of many nontrivial Sobolev maps into a space satisfying (1). Using this theorem together with the results in [33], we can give a partial answer to the question above. Let $(X, d)$ be a metric space, $A \subset \mathbb{R}^{2}$, and $\alpha>0$. A map $\varphi: A \rightarrow X$ is said to be $(L, \alpha)$-Hölder if $d\left(\varphi(a), \varphi\left(a^{\prime}\right)\right) \leq L \cdot\left|a-a^{\prime}\right|^{\alpha}$ for all $a, a^{\prime} \in A$. The space $X$ is said to have the planar $\alpha$-Hölder extension property if there exists $\lambda \geq 1$ such that any $(L, \alpha)$-Hölder map $\varphi: A \rightarrow X$ with $A \subset \mathbb{R}^{2}$ extends to an $(\lambda L, \alpha)$-Hölder map $\bar{\varphi}: \mathbb{R}^{2} \rightarrow X$. Theorem 1.4 together with [33, Theorems 7.1 and 6.4] implies the following.

\section{COROLLARY 1.7}

Let $X$ be a complete, geodesic metric space homeomorphic to $\bar{D}, S^{2}$, or $\mathbb{R}^{2}$. Suppose that there exists $C>0$ such that every Jordan curve in $X$ bounds a Jordan domain $U \subset X$ with

$$
\mathscr{H}^{2}(U) \leq C \cdot \ell(\partial U)^{2} .
$$

Then $X$ has the planar $\alpha$-Hölder extension property for every $\alpha \in(0,1)$.

The following special case provides our almost answer to Question 1.6. 
COROLLARY 1.8

Let $X$ be a geodesic, Ahlfors 2-regular, linearly locally connected metric space homeomorphic to $\bar{D}$ or $S^{2}$. Then for every $\alpha \in(0,1)$ there exists $\lambda \geq 1$ such that if $\varphi: S^{1} \rightarrow X$ is an L-Lipschitz map, then $\varphi$ extends to an $(\lambda L, \alpha)$-Hölder map on all of $\bar{D}$.

The structure of the paper is as follows. In Section 2, we gather definitions and some basic results which will be used throughout the paper. Section 3 contains background from the theory of metric-space-valued Sobolev maps. We furthermore summarize the main existence and regularity results for energy minimizers in metric spaces. Finally, we provide an embeddedness criterion for minimal disks, which is used in our main results. In Section 4, we prove Theorem 1.2. Section 5 is devoted to the proof of Theorem 1.4. We furthermore obtain Theorem 5.8, which gives an almost parameterization result for surfaces as in Theorem 1.4. In Section 6, we prove Theorem 1.1 and an analogous result for spheres (see Theorem 6.2), yielding, in particular, the Bonk-Kleiner theorem.

\section{Preliminaries}

\subsection{Basic definitions and notations}

The Euclidean norm of a vector $v \in \mathbb{R}^{2}$ is denoted by $|v|$, the open unit disk in $\mathbb{R}^{2}$ by

$$
D:=\left\{z \in \mathbb{R}^{2}:|z|<1\right\}
$$

and its closure by $\bar{D}$. Let $(X, d)$ be a metric space. The open ball in $X$ centered at some point $x_{0}$ of radius $r>0$ is denoted by

$$
B\left(x_{0}, r\right)=B_{X}\left(x_{0}, r\right)=\left\{x \in X: d\left(x, x_{0}\right)<r\right\} .
$$

A Jordan curve in $X$ is a subset of $X$ which is homeomorphic to $S^{1}$. Let $\Gamma \subset X$ be a Jordan curve. A continuous map $c: S^{1} \rightarrow \Gamma$ is called a weakly monotone parameterization of $\Gamma$ if $c$ is the uniform limit of homeomorphisms $c_{i}: S^{1} \rightarrow \Gamma$. The length of a curve $c$ in $X$ is denoted by $\ell_{X}(c)$ or simply by $\ell(c)$. A curve $c:[a, b] \rightarrow X$ is called geodesic if $\ell(c)=d(c(a), c(b))$. The metric space $X$ is called geodesic if any pair of points $x, y \in X$ can be joined by a geodesic. If $c:(a, b) \rightarrow X$ is absolutely continuous, then $c$ is metrically differentiable at almost every $t \in(a, b)$; that is, the limit

$$
\left|c^{\prime}(t)\right|:=\lim _{s \rightarrow t} \frac{d(c(s), c(t))}{|t-s|}
$$

exists (see [21], [22]).

Given $m \geq 0$, the $m$-dimensional Hausdorff measure on $X$ is denoted by $\mathscr{H}_{X}^{m}$ or simply by $\mathscr{H}^{m}$ if there is no danger of ambiguity. The normalizing constant in 
the definition of $\mathscr{H}^{m}$ is chosen in such a way that $\mathscr{H}^{m}$ coincides with the Lebesgue measure on Euclidean $\mathbb{R}^{m}$.

The following elementary observation will be used repeatedly throughout the text.

LEMMA 2.1

Let $(X, d)$ be a geodesic metric space homeomorphic to $D$ or $\bar{D}$, and let $\Omega \subset X$ be a Jordan domain such that $\ell(\partial \Omega)<\infty$. Then the length metric $d_{\bar{\Omega}}$ on $\bar{\Omega}$ is finite for any pair of points and has the following properties:

(i) The metrics $d$ and $d_{\bar{\Omega}}$ induce the same topology on $\bar{\Omega}$.

(ii) For every curve $c$ in $\bar{\Omega}$, we have $\ell_{d}(c)=\ell_{d_{\bar{\Omega}}}(c)$.

(iii) For every Borel set $A \subset \bar{\Omega}$, we have $\mathscr{H}_{d}^{2}(A)=\mathscr{H}_{d_{\bar{\Omega}}}^{2}(A)$.

In particular, the space $\left(\bar{\Omega}, d_{\bar{\Omega}}\right)$ is a geodesic metric space homeomorphic to $\bar{D}$.

Proof

Using that $\partial \Omega$ has finite length, we see that $d_{\bar{\Omega}}$ defines a compact topology. Since $d_{\bar{\Omega}} \geq d$, we therefore obtain that the identity map $\iota:\left(\bar{\Omega}, d_{\bar{\Omega}}\right) \rightarrow(\bar{\Omega}, d)$ is a homeomorphism, proving (i). Statement (ii) follows from [6, Proposition 2.3.12]. Since $\iota$ is locally isometric on $\Omega$, we furthermore get (iii).

\subsection{Quasisymmetric homeomorphisms and conformal modulus}

We collect basic definitions concerning quasisymmetric mappings between metric spaces and the modulus of families of curves. We refer to [14], [15], [44], and [17] for more details.

\section{Definition 2.2}

A metric space $X$ is called linearly locally connected if there exists $\lambda \geq 1$ such that for every $x \in X$ and for all $r>0$, every pair of points in $B(x, r)$ can be joined by a continuum in $B(x, \lambda r)$ and every pair of points in $X \backslash B(x, r)$ can be joined by a continuum in $X \backslash B(x, r / \lambda)$.

If $X$ is geodesic, then the first condition in the definition of linear local connectedness is automatically satisfied. By [4, Lemma 2.5], a metric space $X$ homeomorphic to a closed 2-dimensional manifold is linearly locally connected if and only if $X$ is linearly locally contractible: there exists $\lambda \geq 1$ such that every ball $B(x, r) \subset X$ with $0<r<\lambda^{-1} \cdot \operatorname{diam} X$ is contractible in $B(x, \lambda r)$.

\section{Definition 2.3}

A homeomorphism $\varphi: M \rightarrow X$ between metric spaces $M$ and $X$ is said to be quasisymmetric if there exists a homeomorphism $\eta:[0, \infty) \rightarrow[0, \infty)$ such that 


$$
d(\varphi(z), \varphi(a)) \leq \eta(t) \cdot d(\varphi(z), \varphi(b))
$$

for all $z, a, b \in M$ with $d(z, a) \leq t \cdot d(z, b)$.

Quasisymmetric homeomorphisms preserve the doubling property and linear local connectedness. Recall that a metric space $X$ is called doubling if there exists $N \geq 1$ such that every ball of radius $2 r$ in $X$ can be covered by at most $N$ balls of radius $r$. Subsets of Ahlfors regular spaces are, in particular, doubling.

\section{Definition 2.4}

A metric space $X$ is called Ahlfors 2-regular if there exists $L>0$ such that

$$
L^{-1} \cdot r^{2} \leq \mathscr{H}^{2}(B(x, r)) \leq L \cdot r^{2}
$$

for all $x \in X$ and $0<r<\operatorname{diam} X$.

Let $X$ be a metric space and $\Gamma$ a family of curves in $X$. A Borel function $\rho: X \rightarrow$ $[0, \infty]$ is said to be admissible for $\Gamma$ if $\int_{\gamma} \rho \geq 1$ for every locally rectifiable curve $\gamma \in \Gamma$. We refer to [17] for the definition of the path integral $\int_{\gamma} \rho$. The modulus of $\Gamma$ is defined by

$$
\bmod (\Gamma):=\inf _{\rho} \int_{X} \rho^{2} d \mathscr{H}^{2},
$$

where the infimum is taken over all admissible functions for $\Gamma$. Note that throughout this paper, the reference measure on $X$ will always be the 2-dimensional Hausdorff measure. By definition, $\bmod (\Gamma)=\infty$ if $\Gamma$ contains a constant curve. A property is said to hold for almost every curve in $\Gamma$ if it holds for every curve in $\Gamma_{0}$ for some family $\Gamma_{0} \subset \Gamma$ with $\bmod \left(\Gamma \backslash \Gamma_{0}\right)=0$. In the definition of $\bmod (\Gamma)$, the infimum can equivalently be taken over all weakly admissible functions, that is, Borel functions $\rho: X \rightarrow[0, \infty]$ such that $\int_{\gamma} \rho \geq 1$ for almost every locally rectifiable curve $\gamma \in \Gamma$.

\section{THEOREM 2.5}

Let $X$ be a metric space which is homeomorphic to $\bar{D}$ and satisfies, for some $L>0$ and for every $x \in X$ and $r>0$, that

$$
\mathscr{H}^{2}(B(x, r)) \leq L \cdot r^{2} .
$$

Let $Q \geq 1$, and suppose that $u: \bar{D} \rightarrow X$ is a homeomorphism satisfying

$$
\bmod (\Gamma) \leq Q \cdot \bmod (u \circ \Gamma)
$$

for every family $\Gamma$ of curves in $\bar{D}$. Then $u$ is quasisymmetric if and only if $X$ is linearly locally connected. 
Here, $u \circ \Gamma$ denotes the family of curves $u \circ \gamma$ with $\gamma \in \Gamma$. The proof of Theorem 2.5 is a simple variation of the proof of [15, Theorem 4.7], which we provide for completeness in the Appendix. The theorem furthermore holds with $\bar{D}$ replaced by $S^{2}$.

\subsection{Topological preliminaries}

We recall some topological notions and results which we will need in Section 4. For details we refer to [9], [12], and [8].

\section{Definition 2.6}

Let $X$ and $Y$ be metric spaces and $v: X \rightarrow Y$ a continuous map. If $v^{-1}(y)$ is connected for every $y \in Y$, then $v$ is called monotone. If $v^{-1}(y)$ is totally disconnected for every $y \in Y$, then $v$ is called light.

The monotone-light factorization theorem due to Eilenberg and Whyburn (see [51, Theorem 3.5]) asserts that, for every continuous, surjective map $v: X \rightarrow Y$ between compact metric spaces $X$ and $Y$, there exist a compact metric space $Z$ and continuous surjective maps $v_{1}: X \rightarrow Z$ and $v_{2}: Z \rightarrow Y$ such that $v_{1}$ is monotone, $v_{2}$ is light, and $v=v_{2} \circ v_{1}$. The fibers $v_{1}^{-1}(z)$ are exactly the connected components of $v^{-1}\left(v_{2}(z)\right)$.

We will furthermore need the notion of cell-like spaces and cell-like maps (cf. [9, p. 112]).

\section{Definition 2.7}

A compact metric space is called cell-like if it admits an embedding into the Hilbert cube $Q$ in which it is null-homotopic in every neighborhood of itself. A continuous surjection $f: X \rightarrow Y$ between metric spaces $X$ and $Y$ is called cell-like if $f^{-1}(q)$ is cell-like (in particular compact) for every $q \in Y$.

A compact subset $X$ of $S^{2}$ is cell-like if and only if $X$ is connected and $S^{2} \backslash X$ is nonempty and connected. A closed connected subset $X$ of $\bar{D}$ is cell-like if and only if each connected component of $\bar{D} \backslash X$ intersects $\partial D$. A cell-like set contained in a Jordan curve is a point or a topological arc. A compact cell-like metric space $X$ of topological dimension 1 is unicoherent (see [12, p. 332] and [12, p. 97]). Recall that a connected metric space $X$ is unicoherent if, for all closed connected subsets $A, B \subset X$ with $X=A \cup B$, the intersection $A \cap B$ is connected.

Let $X$ and $Y$ be absolute neighborhood retracts. A continuous surjective map $f: X \rightarrow Y$ is cell-like if and only if for every open set $U \subset Y$ the restriction

$$
\left.f\right|_{f^{-1}(U)}: f^{-1}(U) \rightarrow U
$$


is a homotopy equivalence. In particular, if $f$ is cell-like, then, for every open connected (resp., contractible) set $U \subset Y$, the preimage $f^{-1}(U)$ is connected (resp., contractible).

LEMMA 2.8

Let $W \subset S^{2}$ be an open, connected set, and let $K \subset W$ be a compact set all of whose connected components are cell-like. Then $W \backslash K$ is connected. Moreover, for every connected component $T$ of $K$ there exist arbitrarily small neighborhoods $V \subset W$ of $T$ which are homeomorphic to $D$ and such that $\bar{V}$ is homeomorphic to $\bar{D}$ and $\partial V$ does not intersect $K$.

\section{Proof}

Let $Y$ be the space obtained from $W$ by identifying the points in each connected component of $K$, endowed with the finest topology such that the natural projection $\pi: W \rightarrow Y$ is continuous. Then $\pi$ is a cell-like map and hence $Y$ is homeomorphic to $W$ by Moore's theorem (see, e.g., [8, Theorem 25.1] or [9, p. 116]). Moreover, $\pi(K)$ is totally disconnected in $Y$ and hence $Y \backslash \pi(K)$ is connected. It follows that $W \backslash K$ is also connected. This proves the first statement of the lemma.

Let now $T \subset K$ be a connected component of $K$. Then there exist arbitrarily small neighborhoods $U$ of the point $\pi(T) \in Y$ such that $U$ is homeomorphic to $D$, $\bar{U}$ is homeomorphic to $\bar{D}$, and such that the circle $\partial U$ does not intersect the totally disconnected set $\pi(K)$. (In order to construct such $U$, it suffices to find a Jordan curve around the point $\pi(T)$ which avoids the set $\pi(K)$ and let $U$ be the enclosed Jordan domain.) Then $V=\pi^{-1}(U)$ is an arbitrarily small neighborhood of $T$ which is homeomorphic to $D$, such that $\bar{V}$ is homeomorphic to $\bar{D}$ and such that the circle $\partial V$ does not intersect $K$.

\section{PROPOSITION 2.9}

For a continuous surjective map $f: \bar{D} \rightarrow \bar{D}$, the following statements are equivalent:

(i) $f$ is monotone.

(ii) $f$ is cell-like.

(iii) $\quad f$ is a uniform limit of homeomorphisms $f_{i}: \bar{D} \rightarrow \bar{D}$.

\section{Proof}

Clearly, (ii) implies (i). Property (i) is equivalent to (iii) by [50]. Finally, (iii) implies (ii) since the uniform limit of cell-like maps between compact absolute neighborhood retracts is cell-like by [8, Theorem 17.4]. 


\section{Metric-space-valued Sobolev maps}

We recall some definitions from the theory of metric-space-valued Sobolev mappings based on upper gradients; see [16], [42], and [17], as well as the results concerning existence and regularity of energy minimizing disks established in [28], [29], and [30].

Let $(X, d)$ be a complete metric space and $\Omega \subset \mathbb{R}^{2}$ a bounded domain. A Borel function $g: \Omega \rightarrow[0, \infty]$ is said to be an upper gradient of a map $u: \Omega \rightarrow X$ if

$$
d(u(\gamma(a)), u(\gamma(b))) \leq \int_{\gamma} g
$$

for every rectifiable curve $\gamma:[a, b] \rightarrow \Omega$. If (2) only holds for almost every curve $\gamma$, then $g$ is called a weak upper gradient of $u$. A weak upper gradient $g$ of $u$ is called a minimal weak upper gradient of $u$ if $g \in L^{2}(\Omega)$ and if for every weak upper gradient $h$ of $u$ in $L^{2}(\Omega)$ we have $g \leq h$ almost everywhere on $\Omega$.

Denote by $L^{2}(\Omega, X)$ the collection of measurable and essentially separably valued maps $u: \Omega \rightarrow X$ such that the function $u_{x}(z):=d(u(z), x)$ belongs to $L^{2}(\Omega)$ for some and thus any $x \in X$. A map $u \in L^{2}(\Omega, X)$ belongs to the (Newton-) Sobolev space $N^{1,2}(\Omega, X)$ if $u$ has a weak upper gradient in $L^{2}(\Omega)$. Every such map $u$ has a minimal weak upper gradient $g_{u}$, unique up to sets of measure zero (see [17, Theorem 6.3.20]). The Reshetnyak energy of a map $u \in N^{1,2}(\Omega, X)$ is defined by

$$
E_{+}^{2}(u):=\left\|g_{u}\right\|_{L^{2}(\Omega)}^{2} .
$$

If $u \in N^{1,2}(\Omega, X)$, then for almost every $z \in \Omega$ there exists a unique seminorm on $\mathbb{R}^{2}$, denoted by ap md $u_{z}$ and called the approximate metric derivative of $u$, such that

$$
\text { ap } \lim _{y \rightarrow z} \frac{d(u(y), u(z))-\operatorname{apmd} u_{z}(y-z)}{|y-z|}=0
$$

(see [20] and [29, Proposition 4.3]). Here, ap lim denotes the approximate limit (see [10]).

The following notion of parameterized area was introduced in [29].

\section{Definition 3.1}

The parameterized (Hausdorff) area of a map $u \in N^{1,2}(\Omega, X)$ is defined by

$$
\operatorname{Area}(u)=\int_{\Omega} \mathbf{J}\left(\operatorname{apm} \operatorname{mo} u_{z}\right) d z
$$

where the Jacobian $\mathbf{J}(s)$ of a seminorm $s$ on $\mathbb{R}^{2}$ is the Hausdorff 2-measure on $\left(\mathbb{R}^{2}, s\right)$ of the unit square if $s$ is a norm and zero otherwise. 
The area of the restriction of $u$ to a measurable set $B \subset \Omega$ is defined analogously. If $u$ is injective and satisfies Lusin's condition $(\mathrm{N})$, then Area $(u)=\mathscr{H}^{2}(u(\Omega))$ by the area formula (see [20], [21]). For the proof of the following lemma (see [33, Lemma 3.6]).

LEMMA 3.2

Given a Lipschitz curve c : $S^{1} \rightarrow X$, there exists a Lipschitz homotopy of zero parameterized area between $c$ and its constant speed parameterization.

Recall that, by John's theorem, the unit ball with respect to a norm $\|\cdot\|$ on $\mathbb{R}^{2}$ contains a unique ellipse of maximal area, called John's ellipse of $\|\cdot\|$. We will need the following definition from [30].

\section{Definition 3.3}

A map $u \in N^{1,2}(\Omega, X)$ is called infinitesimally isotropic if for almost every $z \in \Omega$ the seminorm ap $\operatorname{md} u_{z}$ is either zero or is a norm and the John ellipse of ap md $u_{z}$ is a Euclidean disk.

We call a map $u \in N^{1,2}(\Omega, X)$ infinitesimally $Q$-quasiconformal if

$$
\left(g_{u}(z)\right)^{2} \leq Q \cdot \mathbf{J}\left(\operatorname{apmd} u_{z}\right)
$$

for almost every $z \in \Omega$. If $u \in N^{1,2}(\Omega, X)$ is infinitesimally isotropic, then it is infinitesimally $Q$-quasiconformal with $Q=\frac{4}{\pi}$ (see [30]).

If $u \in N^{1,2}(D, X)$, then for almost every $v \in S^{1}$ the curve $t \mapsto u(t v)$ with $t \in$ $[1 / 2,1)$ is absolutely continuous. The trace of $u$ is defined by

$$
\operatorname{tr}(u)(v):=\lim _{t \nearrow 1} u(t v)
$$

for almost every $v \in S^{1}$. It can be shown that $\operatorname{tr}(u) \in L^{2}\left(S^{1}, X\right)$ (see [22]). If $u$ is the restriction to $D$ of a continuous map $\hat{u}$ on $\bar{D}$, then $\operatorname{tr}(u)=\left.\hat{u}\right|_{S^{1}}$.

Given a Jordan curve $\Gamma \subset X$, we denote by $\Lambda(\Gamma, X)$ the possibly empty family of maps $v \in N^{1,2}(D, X)$ whose trace has a continuous representative which weakly monotonically parameterizes $\Gamma$. The following theorem summarizes the existence and regularity properties of energy minimizers which we will need in this article and which were proved in [29], [30], and [28]. Note that the results in these papers are stated using a different but equivalent definition of Sobolev mapping and Reshetnyak energy (see [39] and [17, Theorem 7.1.20]).

THEOREM 3.4

Let $X$ be a proper metric space admitting a quadratic isoperimetric inequality. Let 
$\Gamma \subset X$ be a Jordan curve such that $\Lambda(\Gamma, X)$ is not empty. Then there exists $u \in$ $\Lambda(\Gamma, X)$ satisfying

$$
E_{+}^{2}(u)=\inf \left\{E_{+}^{2}(v): v \in \Lambda(\Gamma, X)\right\} .
$$

Any such $u$ is infinitesimally isotropic and has a representative which is continuous on $D$ and extends continuously to $\bar{D}$.

If $X$ is as in the theorem and $\Gamma \subset X$ is a Jordan curve of finite length, then $\Lambda(\Gamma, X)$ is not empty.

\section{Proof}

The existence of an energy minimizer in $\Lambda(\Gamma, X)$ follows from [29, Theorem 7.6]. Continuity of energy minimizers up to the boundary is a consequence of [30, Theorem 4.4] or [28, Theorem 1.3]. Infinitesimal isotropy follows from [30, Lemmas $3.2,4.1]$ (see also [29, Lemma 6.5]).

We next prove the following proposition. See [49, Theorem 1.1] for an analogous result.

PROPOSITION 3.5

Let $X$ be a complete metric space, and let $u: \bar{D} \rightarrow X$ be continuous and monotone. If $u \in N^{1,2}(D, X)$ and $u$ is infinitesimally $Q$-quasiconformal, then

$$
\bmod (\Gamma) \leq Q \cdot \bmod (u \circ \Gamma)
$$

for every family $\Gamma$ of curves in $\bar{D}$.

\section{Proof}

Let $g_{u}$ be the minimal weak upper gradient of $u$ on $D$. We first claim that the upper gradient inequality (2) holds with $g=g_{u}$ for almost every rectifiable curve $\gamma$ in $\bar{D}$ instead of $D$. Indeed, $u$ extends to a Newton-Sobolev map on the open disk $B(0,2) \subset$ $\mathbb{R}^{2}$. An extension can be obtained by reflection along $S^{1}$ and applying the general gluing theorem (see [22, Theorem 1.12.3]). Hence, $u \circ \gamma$ is absolutely continuous for almost every rectifiable curve in $\bar{D}$, parameterized by its arc-length. Since almost every curve $\gamma$ in $\bar{D}$ intersects the boundary $S^{1}$ in a set of Hausdorff 1-measure zero, the claim follows from (2).

Let $\Gamma$ be a family of curves in $\bar{D}$. Then for almost every rectifiable curve $\gamma \in \Gamma$, parameterized by its arc-length, the curve $u \circ \gamma$ is absolutely continuous, and for almost every $t$ we have $\gamma(t) \notin S^{1}$ and $u \circ \gamma$ is metrically differentiable at $t$ with

$$
\left|(u \circ \gamma)^{\prime}(t)\right| \leq g_{u}(\gamma(t))
$$


(see [17, Proposition 6.3.3]). Let $\varrho: X \rightarrow[0, \infty]$ be an admissible function for the family $u \circ \Gamma$, and define $\bar{\varrho}:=g_{u} \cdot(\varrho \circ u)$ on $D$. Then for every curve $\gamma \in \Gamma$ with the properties above, inequality (4) yields

$$
\int_{\gamma} \bar{\varrho}=\int g_{u}(\gamma(t)) \cdot \varrho(u \circ \gamma(t)) d t \geq \int\left|(u \circ \gamma)^{\prime}(t)\right| \cdot \varrho(u \circ \gamma(t)) d t=\int_{u \circ \gamma} \varrho \geq 1,
$$

so $\bar{\varrho}$ is weakly admissible for $\Gamma$.

By [29, Proposition 3.2], there exists a set $A \subset D$ of measure zero such that the restriction $\left.u\right|_{D \backslash A}$ has Lusin's property (N). For $x \in u(D)$, let $N(u, x)$ denote the number of points $z \in D$ with $u(z)=x$. Clearly, $N(u, x)$ equals 1 or $\infty$ for every $x \in u(D)$ because $u$ is monotone. Since

$$
\int_{u(D \backslash A)} N(u, x) d \mathscr{H}^{2}(x)=\int_{D \backslash A} \mathbf{J}\left(\operatorname{apm} \operatorname{md} u_{z}\right) d z=\operatorname{Area}(u)<\infty,
$$

by the area formula (see [20], [21]) it follows that $N(u, x)=1$ for $\mathscr{H}^{2}$-almost every $x \in u(D \backslash A)$. Thus, since $u$ is infinitesimally $Q$-quasiconformal and monotone, it follows again from the area formula that

$$
\int_{D} \bar{\varrho}(z)^{2} d z \leq Q \cdot \int_{D} \varrho \circ u(z)^{2} \cdot \mathbf{J}\left(\operatorname{apmd} u_{z}\right) d z \leq Q \cdot \int_{X} \varrho(x)^{2} d \mathscr{H}^{2}(x) .
$$

Since $\varrho$ was an arbitrary admissible function for $u \circ \Gamma$, it therefore follows that

$$
\bmod (\Gamma) \leq Q \cdot \bmod (u \circ \Gamma)
$$

This completes the proof.

We end this section with the following result needed in the proof of Theorem 6.1.

\section{THEOREM 3.6}

Let $X$ be a complete metric space, and suppose that there exists $L>0$ such that for all $x \in X$ and $r>0$ we have

$$
\mathscr{H}^{2}(B(x, r)) \leq L \cdot r^{2}
$$

Let $u: \bar{D} \rightarrow X$ be continuous, monotone, and nonconstant. If $u \in N^{1,2}(D, X)$ and $u$ is infinitesimally quasiconformal, then $u$ is a homeomorphism onto its image.

\section{Proof}

It suffices to show that $u$ is injective. We argue by contradiction and suppose that there exists $x \in u(\bar{D})$ such that $E:=u^{-1}(x)$ consists of more than one point. Fix $r>0$ such that $F_{r}:=\bar{D} \backslash u^{-1}(B(x, r))$ is not empty. Note that such $r$ exists since $u$ 
is assumed to be nonconstant. Let $\Gamma:=\Gamma\left(E, F_{r} ; \bar{D}\right)$ denote the family of curves in $\bar{D}$ joining $E$ and $F_{r}$.

We first show that the modulus of $\Gamma$ is bounded from above independently of $r$. Let $Q \geq 1$ be such that $u$ is infinitesimally $Q$-quasiconformal. By Proposition 3.5, we have

$$
\bmod (\Gamma) \leq Q \cdot \bmod (u \circ \Gamma)
$$

Since $u \circ \Gamma$ is a subset of the family of curves in $X$ joining $\bar{B}(x, r / 4)$ to $X \backslash B(x, r)$, it follows from [14, Lemma 7.18] that $\bmod (u \circ \Gamma)$ is bounded by a constant $L^{\prime}$ only depending on $L$. This shows that $\bmod (\Gamma) \leq Q L^{\prime}$.

Since $E$ is a nondegenerate continuum (i.e., consisting of more than one point), this bound on the modulus is easily seen to contradict the Loewner property of $\bar{D}$. Indeed, let $z_{0} \in \bar{D} \backslash E$ and let $z_{1} \in E$ be a point on $E$ nearest to $z_{0}$. For $s>0$ sufficiently small, let $G_{s}$ denote the set of points on the straight segment from $z_{0}$ to $z_{1}$ which are at least a distance $s$ away from $E$. Then $G_{s}$ is a nondegenerate continuum and, for $s>0$ sufficiently small, we have

$$
\operatorname{dist}\left(E, G_{s}\right) \leq C \cdot s \cdot \min \left\{\operatorname{diam} E, \operatorname{diam} G_{s}\right\}
$$

for some $C$ not depending on $s$. Fix $s$ as above, and let $r>0$ be so small that $G_{s} \subset F_{r}$, where $F_{r}$ is as at the beginning of the proof. Then $\Gamma\left(E, F_{r} ; \bar{D}\right)$ contains the family $\Gamma\left(E, G_{s} ; \bar{D}\right)$ of curves in $\bar{D}$ connecting $E$ and $G_{s}$ and hence

$$
\bmod \left(\Gamma\left(E, G_{s} ; \bar{D}\right)\right) \leq \bmod \left(\Gamma\left(E, F_{r} ; \bar{D}\right)\right) \leq Q L^{\prime}
$$

for all $s>0$ sufficiently small. However, this is impossible since

$$
\bmod \left(\Gamma\left(E, G_{s} ; \bar{D}\right)\right) \rightarrow \infty
$$

as $s \rightarrow 0^{+}$by the 2-Loewner property of $\bar{D}$ (see, e.g., [14, Theorem 8.23 and Example 8.24]). This completes the proof.

\section{Topological properties of energy minimizers}

In this section we prove Theorem 1.2. The key ingredient is the following topological result.

\section{THEOREM 4.1}

Let $X$ be a geodesic metric space homeomorphic to $\bar{D}$. Let $v: \bar{D} \rightarrow X$ be a continuous and surjective map satisfying the following properties:

(i) The restriction of $v$ to $S^{1}$ is a weakly monotone parameterization of $\partial X$.

(ii) Whenever $T \subset X$ is a single point or bi-Lipschitz homeomorphic to a closed interval, every connected component of $v^{-1}(T)$ is cell-like.

Then $v$ is a cell-like map. 
Before proving Theorem 4.1, we first provide the following.

\section{Proof of Theorem 1.2}

Let $u$ be as in the theorem. Then $u$ is infinitesimally quasiconformal by Theorem 3.4.

Notice that $u$ need not minimize the parameterized Hausdorff area among all maps in $\Lambda(\partial X, X)$; however, it minimizes a somewhat different area functional. Indeed, by [30, Theorem 1.1], the map $u$ minimizes the inscribed Riemannian area

$$
\operatorname{Area}_{\mu^{i}}(u):=\int_{D} \mathbf{J}_{\mu^{i}}\left(\operatorname{ap} \operatorname{md} u_{z}\right) d z
$$

among all maps in $\Lambda(\partial X, X)$, where the $\mu^{i}$-jacobian $\mathbf{J}_{\mu^{i}}(s)$ of a seminorm $s$ on $\mathbb{R}^{2}$ is defined as follows. If $s$ is degenerate, then $\mathbf{J}_{\mu^{i}}(s)=0$; if $s$ is a norm, then $\mathbf{J}_{\mu^{i}}(s)=$ $\frac{\pi}{|L|}$, where $|L|$ is the Lebesgue measure of John's ellipse of $\left\{v \in \mathbb{R}^{2}: s(v) \leq 1\right\}$.

Due to Proposition 2.9, in order to prove the theorem it is enough to show that $u$ satisfies the hypotheses of Theorem 4.1. For topological reasons, the map $u$ is surjective and satisfies property (i). In order to see that $u$ also satisfies property (ii), we argue by contradiction and assume that there exists $T$ as in (ii) such that some connected component $K$ of $u^{-1}(T)$ is not cell-like. Thus, there exists a connected component of $\bar{D} \backslash K$ which does not intersect $S^{1}$. In particular, there also exists a connected component $U$ of $\bar{D} \backslash u^{-1}(T)$ which does not intersect $S^{1}$. Since $T$ is a single point or bi-Lipschitz homeomorphic to a closed interval, it follows that $T$ is an absolute Lipschitz retract (see [2, Proposition I.1.2]). Therefore, there exists a Lipschitz retraction $P: X \rightarrow T$. Define a map by $w:=P \circ u$.

We claim that the restrictions $\left.w\right|_{U}$ and $\left.u\right|_{U}$ have the same trace in the sense of [31, Definition 4.1]. Indeed, for fixed $x \in X$ consider the real-valued function $h(z):=d(x, u(z))-d(x, w(z))$ for $z \in \bar{U}$, where $d$ is the metric on $X$. Then $h$ belongs to $W^{1,2}(U)$, is continuous on $\bar{U}$, and satisfies $h=0$ on $\partial U$. Since $h$ extends continuously to $\mathbb{R}^{2}$ with the value 0 on $\mathbb{R}^{2} \backslash U$, it follows directly from the HavinBagby theorem (see, e.g., [13, Theorem 2.1]) that $h \in W_{0}^{1,2}(U)$. Since $x$ was arbitrary, this shows that $\left.u\right|_{U}$ and $\left.w\right|_{U}$ have equal traces in the sense of [31, Definition 4.1].

By [31, Lemma 4.2], we may replace $\left.u\right|_{U}$ by $\left.w\right|_{U}$ and obtain another map $u_{1} \in$ $N^{1,2}(D, X)$ with the same trace as $u$ and, in particular, $u_{1} \in \Lambda(\partial X, X)$. Since $T$ is a single point or bi-Lipschitz homeomorphic to a closed interval, it follows that ap md $w_{z}$ is degenerate for almost every $z$ and hence the inscribed Riemannian area of $w$ is zero. Since $u$ minimizes the inscribed Riemannian area, it follows that the inscribed Riemannian area of $\left.u\right|_{U}$ is zero. Since $u$ is infinitesimally quasiconformal, the Reshetnyak energy of $\left.u\right|_{U}$ must be zero as well. Therefore $\left.u\right|_{U}$ is constant and hence $u(U)$ is contained in $T$, a contradiction. Thus every connected component of $u^{-1}(T)$ is cell-like and hence $u$ satisfies (ii). This completes the proof. 
The following result will be used in the proof of Theorem 4.1 and later.

\section{LEMMA 4.2}

Let $X$ be a geodesic metric space, and let $\Gamma \subset X$ be a topological arc connecting two points $a, b \in X$. Then for every $\varepsilon>0$ there exists a bi-Lipschitz curve contained in the $\varepsilon$-neighborhood of $\Gamma$ and connecting $a$ and $b$.

A similar statement holds for Jordan curves.

\section{Proof}

Let $\varepsilon>0$ and choose a piecewise geodesic curve $\gamma$ which is contained in the $\frac{\varepsilon}{2}$ neighborhood of $\Gamma$ and connects the endpoints $a$ and $b$ of $\Gamma$. By choosing an appropriate subcurve of $\gamma$, we may assume that $\gamma$ is an injective piecewise bi-Lipschitz curve. By changing the curve step by step near its vertices, it suffices to prove the following claim.

\section{CLAIM 4.3}

Let $s>0$, and let $\eta:[-s, s] \rightarrow X$ be an injective curve such that the restrictions $\left.\eta\right|_{[0, s]}$ and $\left.\eta\right|_{[-s, 0]}$ are geodesics parameterized by their arc-length. Then there exist arbitrarily small $t \in(0, s)$ such that after replacing $\left.\eta\right|_{[-t, t]}$ by a geodesic from $\eta(-t)$ to $\eta(t)$ we obtain a bi-Lipschitz curve.

To prove the claim, note first that the Lipschitz function $f(t)=d(\eta(-t), \eta(t))$ satisfies $f(0)=0$ and is strictly positive for $t>0$. Thus we find arbitrarily small $t>0$ for which $f^{\prime}(t)$ exists and is strictly positive. Fix such $t$, and set $\delta:=f^{\prime}(t)$. Choose a geodesic $c_{t}$ from $\eta(-t)$ to $\eta(t)$, parameterized by its arc-length and such that $c_{t}(0)=\eta(-t)$. The triangle inequality and the fact that $f^{\prime}(t)=\delta>0$ yield that, for all sufficiently small $r>0$, we have

$$
d\left(\eta(-t-r), c_{t}(r)\right) \geq \frac{\delta \cdot r}{2} .
$$

Since this holds for every such geodesic $c_{t}$ it follows that $c_{t}$ can only intersect $\left.\eta\right|_{[-s,-t]}$ at $\eta(-t)$. Moreover, the inequality above together with the triangle inequality imply that the concatenation of $\left.\eta\right|_{[-s,-t]}$ with $c_{t}$ is a bi-Lipschitz curve locally around $\eta(-t)$ and hence also globally. The same argument shows that the concatenation of $c_{t}$ with $\left.\eta\right|_{[t, s]}$ is a bi-Lipschitz curve, and hence the concatenation of all three curves is biLipschitz. This proves the claim and completes the proof of the lemma.

The rest of this section is devoted to the proof of Theorem 4.1. Let $v$ be as in the statement of the theorem. We first claim that it is enough to consider the case that $v$ is, 
in addition, a light map. Indeed, by the monotone-light factorization theorem, there exist a metric space $Z$ and continuous, surjective maps $v_{1}: \bar{D} \rightarrow Z$ and $v_{2}: Z \rightarrow X$ such that $v_{1}$ is monotone, $v_{2}$ is light, and $v$ factors as $v=v_{2} \circ v_{1}$. Moreover, the fibers $v_{1}^{-1}(z)$ are exactly the connected components of $v^{-1}\left(v_{2}(z)\right)$. It thus follows from the properties of $v$ that $v_{1}$ and the restriction $\left.v_{1}\right|_{S^{1}}$ are cell-like maps. Consequently, $Z$ is homeomorphic to $\bar{D}$ and $v_{1}$ is the uniform limit of homeomorphisms (see [31, Corollary 7.12]). We now identify $Z$ with $\bar{D}$ via a homeomorphism and show the following.

LEMMA 4.4

The map $v_{2}$ satisfies properties (i) and (ii) of Theorem 4.1 .

\section{Proof}

We first prove that $v_{2}$ has property (i). As mentioned above, we identify $Z$ with $\bar{D}$ via a homeomorphism. Since $v_{1}$ is the uniform limit of homeomorphisms, it follows that $v_{1}\left(S^{1}\right)=S^{1}$ and hence $v_{2}\left(S^{1}\right)=\partial X$. Let $x \in \partial X$. We show that $v_{2}^{-1}(x) \cap$ $S^{1}$ consists of a single point. Let $z, z^{\prime} \in S^{1}$ be such that $v_{2}(z)=x=v_{2}\left(z^{\prime}\right)$. The preimages $v_{1}^{-1}(z)$ and $v_{1}^{-1}\left(z^{\prime}\right)$ are connected components of $v^{-1}(x)$, both having nontrivial intersection with $S^{1}$. Since $v^{-1}(x) \cap S^{1}$ is an interval or a point, we must therefore have $z=z^{\prime}$. This proves property (i).

As for property (ii), let $T \subset X$ be a point or bi-Lipschitz homeomorphic to a closed interval and let $K \subset \bar{D}$ be a connected component of $v_{2}^{-1}(T)$. We must show that $K$ is cell-like. Since $v_{1}$ is surjective and monotone, we have that $K^{\prime}:=v_{1}^{-1}(K)$ is connected and thus $K^{\prime}$ is a connected component of $v^{-1}(T)$. In particular, $K^{\prime}$ is cell-like and hence $K$ is cell-like as well by [24, Theorem 1.4]. This proves that $v_{2}$ satisfies property (ii) and completes the proof of the lemma.

Now, if $v_{2}$ is cell-like, then so is $v$ because $v_{1}$ is cell-like. This together with Lemma 4.4 shows our claim. Thus, for the proof of Theorem 4.1 it is indeed enough to consider only the case that $v$ is, in addition, a light map. We henceforth assume that $v$ is as in the statement of Theorem 4.1 and that $v$ is also light. We must show that $v$ is injective. For this, we first prove some auxiliary results.

LEMMA 4.5

For every $x \in \partial X$ the preimage $v^{-1}(x)$ consists of exactly one point.

\section{Proof}

Since $v$ is light and its restriction to $S^{1}$ is a weakly monotone parameterization of $\partial X$, the point $x$ has exactly one preimage in $S^{1}$. We must show that $x$ has no preim- 
ages in $D$. We argue by contradiction and assume that there exists $z \in D$ such that $v(z)=x$. There exists an open neighborhood $U \subset D$ of $z$ homeomorphic to $D$ such that $\bar{U}$ is homeomorphic to $\bar{D}$ and $\partial U$ does not intersect the totally disconnected set $v^{-1}(x)$. We find an open neighborhood $W$ of $x$ which does not intersect $v(\partial U)$. We then find an $\operatorname{arc} S$ in $W$ separating $x$ from $v(\partial U)$; just take $S$ to be any arc in $W$ which intersects $\partial X$ exactly in the two boundary points of $S$ lying on "different sides" of $x$. By Lemma 4.2 we may assume that $S$ is a bi-Lipschitz curve. Since $S$ separates $v(U)$, the set $v^{-1}(S)$ must separate $U$. However, $v^{-1}(S) \cap \partial U=\emptyset$ and any connected component of $v^{-1}(S)$ is cell-like by assumption. Hence the set $K:=v^{-1}(S) \cap U$ is compact and all its connected components are cell-like. So, $U \backslash v^{-1}(S)$ is connected by Lemma 2.8. However, this contradicts the fact that $v^{-1}(S)$ separates $U$ and completes the proof.

Statements similar to the following lemma have been studied under the additional assumption that the map is open (see, e.g., [11]).

\section{LEMMA 4.6}

Let $T \subset X$ be bi-Lipschitz homeomorphic to a closed interval. Then $v(C)=T$ for every connected component $C$ of $v^{-1}(T)$.

\section{Proof}

We argue by contradiction and assume that there exists a connected component $C$ of $v^{-1}(T)$ such that $v(C) \neq T$, and thus $v(C)$ is a compact subarc of $T$. After possibly replacing $T$ by a nontrivial subarc which intersects $v(C)$ exactly in one point, and replacing $C$ by a connected component in $C$ of the preimage of this subarc, we may assume that $v(C)$ is just one endpoint of $T$, which we call $p$. Since $v$ is a light map it follows that $C$ consists of a single point $z \in \bar{D}$.

We first assume that $z \notin S^{1}$. By Lemma 2.8 , there exists an arbitrarily small open neighborhood $U \subset D$ of $z$ homeomorphic to $D$ such that $\bar{U}$ is homeomorphic to $\bar{D}$ and $\partial U$ does not intersect $v^{-1}(T)$. Let $T^{\prime}$ be a nontrivial compact subarc of $T \backslash\{p\}$. Choosing $U$ sufficiently small, we may assume that $T^{\prime}$ has no preimage in $\bar{U}$.

We find an arc $S$ in an arbitrary small neighborhood of $T$ which connects two different points on $T^{\prime}$ and which together with the corresponding part of $T^{\prime}$ defines a Jordan curve $\Gamma$ which separates $v(U)$. Choosing $S$ sufficiently close to $T$, we may assume that $S$ does not intersect $v(\partial U)$. Using Lemma 4.2, we may furthermore assume that $S$ is a bi-Lipschitz curve. Since $T^{\prime}$ does not have any preimage in $\bar{U}$, it follows that the preimage of $\Gamma$ in $\bar{U}$ coincides with the preimage of $S$ in $\bar{U}$. Moreover, this preimage does not intersect $\partial U$. Thus, the set $K:=v^{-1}(\Gamma) \cap U$ is compact and every connected component of $K$ is cell-like, and hence $U \backslash v^{-1}(\Gamma)$ is connected by 
Lemma 2.8. This, however, is impossible since $\Gamma$ separates $v(U)$. This contradiction finishes the proof in the case that $z \notin S^{1}$.

The proof in the case $z \in S^{1}$ is analogous and is left to the reader.

Using the lemmas above we now show that $v$ is injective, which will complete the proof of Theorem 4.1. Let $x \in X$. If $x \in \partial X$, then $v^{-1}(x)$ consists of a single point by Lemma 4.5, so we may assume that $x \notin \partial X$. We first suppose that $x$ is contained in a bi-Lipschitz arc $T \subset X$ with endpoints $x_{ \pm} \in \partial X$. By Lemma 4.5, the two points $x_{ \pm}$have unique preimage points $z_{ \pm} \in S^{1}$. By Lemma 4.6, any connected component of $v^{-1}(T)$ must contain both points $z_{ \pm}$and hence the set $\Gamma=v^{-1}(T)$ is connected. Thus, by assumption (ii) of our theorem, $\Gamma$ must be cell-like. Since $v$ is a light map, the set $\Gamma$ is 1-dimensional (see [12, p. 311]), and hence $\Gamma$ is unicoherent. Now, let $T^{+}$and $T^{-}$be the subintervals into which $x$ subdivides $T$. As above, the preimages $\Gamma^{ \pm}$of $T^{ \pm}$must be connected. Since $\Gamma$ is unicoherent, the intersection $\Gamma^{+} \cap \Gamma^{-}$is connected. Since this intersection is exactly the totally disconnected fiber $v^{-1}(x)$, it follows that $v^{-1}(x)$ has exactly one point.

Now, let $x \in X \backslash \partial X$ be arbitrary. Connect $x$ by a geodesic $S$ with a point on $\partial X$. The construction used in Lemma 4.2 shows that any part of $S$ which does not contain $x$ can be extended to a bi-Lipschitz arc $T$ connecting two points on $\partial X$. Thus, for any point $y$ on $S \backslash\{x\}$, the preimage of $y$ contains only one point. Since $v^{-1}(x)$ is totally disconnected, we deduce from Lemma 4.6 that $v^{-1}(x)$ has also only one point. This shows that $v$ is injective and completes the proof of Theorem 4.1.

\section{A quadratic isoperimetric inequality for metric surfaces}

In this section, we prove Theorem 1.4 from the Introduction, as well as Corollary 5.5 below. In Theorem 5.8, moreover, we obtain an almost parameterization result for spaces as in Theorem 1.4. We begin with the following result, which proves the first part of Theorem 1.4.

\section{THEOREM 5.1}

Let $X$ be a complete, geodesic metric space homeomorphic to $\bar{D}, S^{2}$, or $\mathbb{R}^{2}$. Suppose that there exists $C$ such that every Jordan curve $\Gamma \subset X$ bounds a Jordan domain $\Omega \subset X$ with

$$
\mathscr{H}^{2}(\Omega) \leq C \cdot \ell(\Gamma)^{2}
$$

Then $X$ admits a quadratic isoperimetric inequality with isoperimetric constant only depending on $C$. 
We need some preparations. Recall that a metric space $Y$ is said to be L-Lipschitz 1 -connected up to some scale if there exists $\lambda_{0}>0$ such that every $\lambda$-Lipschitz curve $c: S^{1} \rightarrow Y$ with $\lambda \leq \lambda_{0}$ extends to an $L \lambda$-Lipschitz map $\varphi: \bar{D} \rightarrow Y$.

\section{PROPOSITION 5.2}

Let $X$ be as in Theorem 5.1, and suppose that $Y$ is a metric space which contains $X$ and which is L-Lipschitz 1-connected up to some scale. Then every injective Lipschitz curve c: $S^{1} \rightarrow X$ extends to a Lipschitz map $\varphi: \bar{D} \rightarrow Y$ with

$$
\operatorname{Area}(\varphi) \leq C^{\prime} \cdot \ell(c)^{2}
$$

for some constant $C^{\prime}$ only depending on $C$ and $L$.

Notice that we do not impose a bound on the length of the curve $c$ and that the constant $C^{\prime}$ is independent of the scale up to which $Y$ is Lipschitz 1-connected.

\section{Proof}

Let $c: S^{1} \rightarrow X$ be an injective Lipschitz curve. Then $c$ is homotopic to its constant speed parameterization via a Lipschitz homotopy of zero area by Lemma 3.2. Therefore, we may assume that $c$ is parameterized proportional to its arc-length. Let $\Omega \subset X$ be the Jordan domain of smallest area and boundary $c$. It follows that $\mathscr{H}^{2}(U) \leq C \cdot \ell(\partial U)^{2}$ for every Jordan domain $U \subset \Omega$.

Denote by $d_{\bar{\Omega}}$ the length metric on $\bar{\Omega}$, and set $Z:=\left(\bar{\Omega}, d_{\bar{\Omega}}\right)$. By Lemma 2.1 , the space $Z$ is geodesic and homeomorphic to $\bar{D}$. Moreover, the length of the boundary circle $\partial Z$ as well as the Hausdorff 2-measure of $Z$ are finite. Finally, for every Jordan domain $U \subset Z$, we have $\mathscr{H}_{Z}^{2}(U) \leq C \cdot \ell_{Z}(\partial U)^{2}$.

Let $n \in \mathbb{N}$ be sufficiently large, to be determined later. By [33, Theorem 4.1], there exists a triangulation $\tau$ of $Z$ consisting of at most $K \cdot n^{2}$ triangles of $d \bar{\Omega}$-diameter at most $\frac{\ell(c)}{n}$ each, and such that every edge contained in $\partial Z$ has length at most $\frac{\ell(c)}{n}$. Here, $K$ only depends on $C$. By a triangulation of $Z$, we mean a homeomorphism from $Z$ to a simplicial 2-complex $\tau$. We endow $Z$ with the induced simplicial structure from $\tau$. For $i=0,1,2$ the $i$-skeleton of $\tau$ will be denoted $\tau^{(i)}$ and may thus be viewed as a subset of $Z$. The 2-simplices of $\tau$ will also be called triangles in $\tau$.

Put on each triangle in $\tau$ a piecewise Euclidean metric which makes it an equilateral Euclidean triangle of side-length 1 . Let $d_{\tau}$ denote the resulting length metric on $\tau$. Then $\Sigma:=\left(\tau, d_{\tau}\right)$ is bi-Lipschitz homeomorphic to $\bar{D}$ by [27, Theorem 8.6]. Denote by $P: Z \rightarrow X$ the natural inclusion and observe that $P$ is 1-Lipschitz and a homeomorphism onto $\bar{\Omega}$.

We construct a Lipschitz map $\psi: \Sigma \rightarrow Y$ as follows. For each $v \in \tau^{(0)}$ we set $\psi(v):=P(v)$, where we have identified $v$ with its image in $Z$. Let now $e=[v, w] \in$ 
$\tau^{(1)}$ be an edge. If $e$ is contained in the boundary circle $\partial \Sigma$, then we let $\left.\psi\right|_{e}$ be a constant speed parameterization of the part of $c$ between the points $\psi(v)$ and $\psi(w)$. Otherwise, let $\left.\psi\right|_{e}$ be a constant speed geodesic from $\psi(v)$ to $\psi(w)$ in $X$. It follows that, for every triangle $F \in \tau^{(2)}$, the map $\left.\psi\right|_{\partial F}$ is $M \ell(c) n^{-1}$-Lipschitz, where $M$ is a universal constant. Hence, if $n$ was chosen sufficiently large, then there exists an $M^{\prime} \ell(c) n^{-1}$-Lipschitz extension $\left.\psi\right|_{F}: F \rightarrow Y$ for some $M^{\prime}$ only depending on $L$. Since $F \in \tau^{(2)}$ was arbitrary, this yields a Lipschitz map defined on all of $\Sigma$.

We finally choose a bi-Lipschitz homeomorphism $\varrho: \bar{D} \rightarrow \Sigma$ in such a way that the composition $\psi \circ \varrho$ provides an extension of the curve $c$. For this, let $\varrho: S^{1} \rightarrow \partial \Sigma$ be the piecewise constant speed map such that $\psi \circ \varrho=c$. Then, $\varrho$ is bi-Lipschitz and thus extends to a bi-Lipschitz homeomorphism $\varrho: \bar{D} \rightarrow \Sigma$ by [43, Theorem A] and by the fact (mentioned above) that $\Sigma$ is bi-Lipschitz homeomorphic to $\bar{D}$. Thus, the $\operatorname{map} \varphi:=\psi \circ \varrho$ is Lipschitz and is an extension of $c$. Moreover,

$$
\operatorname{Area}(\varphi)=\sum_{F \in \tau^{(2)}} \operatorname{Area}\left(\left.\psi\right|_{F}\right) \leq \frac{\sqrt{3} K}{4} \cdot n^{2} \cdot\left(M^{\prime} \cdot \frac{\ell(c)}{n}\right)^{2}=C^{\prime} \cdot \ell(c)^{2}
$$

for some constant $C^{\prime}$ only depending on $C$ and $L$. This concludes the proof.

The next lemma will allow us to pass from noninjective Lipschitz curves to injective ones in the proof of Theorem 5.1. For $m \geq 0$, let $A_{m}$ be the closed unit disk $\bar{D}$ with $m$ pairwise-separated open Euclidean disks removed. More precisely,

$$
A_{m}=\bar{D} \backslash \bigcup_{k=1}^{m} D_{k}
$$

for some open Euclidean disks $D_{k}$ such that $\bar{D}_{k} \subset D$ are pairwise disjoint. Up to bi-Lipschitz equivalence, the choice of omitted Euclidean disks does not matter.

\section{LEMMA 5.3}

Let $X$ be a geodesic metric space, and let $c: S^{1} \rightarrow X$ be a Lipschitz curve. Given points $s_{0}, \ldots, s_{N} \in S^{1}$, there exist $m \geq 0$ and a Lipschitz map $\varphi: A_{m} \rightarrow X$ of zero area satisfying the following properties:

(i) $\left.\varphi\right|_{S^{1}}$ is a piecewise geodesic with $\varphi\left(s_{j}\right)=c\left(s_{j}\right)$ for $j=0, \ldots, N$.

(ii) Each curve $\left.\varphi\right|_{\partial D_{k}}$ is a Jordan curve and

$$
\sum_{k=1}^{m} \ell\left(\left.\varphi\right|_{\partial D_{k}}\right) \leq \ell(c)
$$

By a piecewise geodesic we mean the concatenation of finitely many geodesics, where we interpret constant curves as geodesics as well. 


\section{Proof}

We may assume that the points $s_{0}, \ldots, s_{N} \in S^{1}$ are in cyclic order, and we set $s_{N+1}=$ $s_{0}$. Proceeding by induction on $i$, we find curves $\gamma_{i}:\left[s_{0}, s_{i}\right] \rightarrow X$ with the following properties. The restriction $\left.\gamma_{i}\right|_{\left[s_{k-1}, s_{k}\right]}$ is a geodesic between $c\left(s_{k-1}\right)$ and $c\left(s_{k}\right)$ for any $1 \leq k \leq i$, and the image $\gamma_{i}\left(\left[s_{0}, s_{i}\right]\right)$ is a finite topological graph $G_{i}$ in $X$ with geodesic edges. In the inductive step, we first choose an arbitrary geodesic $\eta$ from $c\left(s_{i}\right)$ to $c\left(s_{i+1}\right)$. After possibly modifying $\eta$ on finitely many intervals, we may assume that $\eta$ intersects any edge of $G_{i}$ only at boundary points or connected subsets. Therefore the union of $G_{i}$ and the image of $\eta$ is a finite graph which has geodesic edges. We define $\gamma_{i+1}$ to be the concatenation of $\gamma_{i}$ and $\eta$.

For $i=N+1$ we obtain a piecewise geodesic $\gamma: S^{1} \rightarrow X$ with $\gamma\left(s_{i}\right)=c\left(s_{i}\right)$ for all $i$ and such that $\gamma\left(S^{1}\right)$ is a finite graph $G$ with geodesic edges. Moreover, by construction $\ell(\gamma) \leq \ell(c)$. We may parameterize $\gamma$ to be Lipschitz continuous.

Using topological arguments in the finite graph $G$, we can extend the curve $\gamma$ to a Lipschitz-continuous map $\varphi: A_{m} \rightarrow G$ for some $m$ such that (ii) holds true. Since $\varphi$ has its image in $G$, the area of $\varphi$ is 0 . Let us briefly sketch the construction of this map $\varphi$. Notice first that $\gamma$ is Lipschitz-homotopic in $G$ to a curve $\bar{\gamma}$ of length at most $\ell(\gamma)$ which is either constant or locally injective. If $\bar{\gamma}$ is constant, then the Lipschitz homotopy gives rise to a Lipschitz map $\varphi: A_{0}=\bar{D} \rightarrow G$ such that $\left.\varphi\right|_{S^{1}}=\gamma$. If $\bar{\gamma}$ is globally injective, then the Lipschitz homotopy gives rise to a Lipschitz map $\varphi: A_{1}=$ $\bar{D} \backslash D_{1} \rightarrow G$ such that $\left.\varphi\right|_{S^{1}}=\gamma$ and $\left.\varphi\right|_{\partial D_{1}}=\bar{\gamma}$. In both these cases we are done. Suppose now that $\bar{\gamma}$ is locally injective but not globally injective, and let $I \subset S^{1}$ be a maximal open segment on which $\bar{\gamma}$ is injective. Let $D_{1}$ and $D_{1}^{\prime}$ be two open separated Euclidean disks in $D$. Then $\gamma$ extends to a Lipschitz map $\varphi: \bar{D} \backslash\left(D_{1} \cup D_{1}^{\prime}\right) \rightarrow G$ such that $\left.\varphi\right|_{\partial D_{1}}$ parameterizes the Jordan curve $\left.\bar{\gamma}\right|_{\bar{I}}$ and $\left.\varphi\right|_{\partial D_{1}^{\prime}}$ parameterizes the closed curve $\left.\bar{\gamma}\right|_{S^{1} \backslash I}$. Now, we apply the same construction to the curve $\gamma^{\prime}:=\left.\varphi\right|_{\partial D_{1}^{\prime}}$. After finitely many iterations of this construction we arrive at the desired map.

Let $X$ and $Y$ be metric spaces, and let $\varepsilon>0$. We say that $Y$ is an $\varepsilon$-thickening of $X$ if there exists an isometric embedding $\iota: X \rightarrow Y$ such that the Hausdorff distance between $\iota(X)$ and $Y$ is at most $\varepsilon$. The following lemma, which was proved in [46] and appeared in [33, Lemma 3.3] in its present form, asserts the existence of $\varepsilon$-thickenings with good properties.

\section{LEMMA 5.4}

Let $X$ be a length space. There is a universal constant $M$ such that for every $\varepsilon>0$ there exists a complete length space $X_{\varepsilon}$ which is an $\varepsilon$-thickening of $X$ and has the following property. Let $\lambda>0$, and let $c_{0}: S^{1} \rightarrow X_{\varepsilon}$ be $\lambda$-Lipschitz. If $\lambda \leq \frac{\varepsilon}{M}$, then $c_{0}$ is $M \lambda$-Lipschitz homotopic to a constant curve. If $\lambda \geq \frac{\varepsilon}{M}$, then $c_{0}$ is Lipschitz 
homotopic to a curve $c_{1}: S^{1} \rightarrow X$ with $\ell\left(c_{1}\right) \leq 2 \ell(c)$ via a homotopy of area at most $M \varepsilon \lambda$. Furthermore, if $X$ is locally compact, then $X_{\varepsilon}$ is locally compact.

In particular, $X_{\varepsilon}$ is Lipschitz 1-connected up to some scale. We can now provide the following.

\section{Proof of Theorem 5.1}

Let $k \geq 2$, and let $X_{k}$ be a $\frac{1}{k}$-thickening of $X$ as in Lemma 5.4. Note that $X_{k}$ is locally compact, geodesic, and converges in the Gromov-Hausdorff sense to $X$ as $k \rightarrow \infty$. By [33, Theorem 1.8], a quadratic isoperimetric inequality in the sense of Definition 1.3 is stable under taking Gromov-Hausdorff limits. In order to prove Theorem 5.1, it thus suffices to show that each $X_{k}$ admits a quadratic isoperimetric inequality with isoperimetric constant depending only on $C$.

Fix $k \geq 2$, and let $c: S^{1} \rightarrow X_{k}$ be a Lipschitz curve. We will show that $c$ extends to a Lipschitz map $\varphi: \bar{D} \rightarrow X_{k}$ of area at most $C^{\prime} \cdot \ell(c)^{2}$, where $C^{\prime}$ only depends on $C$. By Lemma 3.2, we may assume that $c$ has constant speed. By Lemma 5.4, there exists a Lipschitz homotopy $\varphi_{1}$ of area

$$
\operatorname{Area}\left(\varphi_{1}\right) \leq M \cdot \ell(c)^{2}
$$

from $c$ to a Lipschitz curve $c_{1}$ which is either constant or has image in $X$ and satisfies $\ell\left(c_{1}\right) \leq 2 \ell(c)$. Here, $M$ denotes a suitable universal constant. If $c_{1}$ is constant, then we are done, so we may assume $c_{1}$ to be nonconstant and to have image in $X$. As above, we may assume that $c_{1}$ has constant speed.

Fix $N \in \mathbb{N}$ sufficiently large (see below), and let $s_{0}, \ldots, s_{N} \in S^{1}$ be equidistant points in cyclic order. Let $\varphi_{2}: A_{m} \rightarrow X$ be a Lipschitz map of zero area as in Lemma 5.3, when applied to the curve $c_{1}$. Set $c_{2}:=\left.\varphi_{2}\right|_{S^{1}}$, and note that

$$
\ell\left(c_{2}\right) \leq \ell\left(c_{1}\right) \leq 2 \ell(c) .
$$

Set $s_{N+1}:=s_{0}$. If $N$ was chosen sufficiently large, then there exist Lipschitz homotopies in $X_{k}$ of area at most $M^{\prime} \cdot \frac{\ell\left(c_{1}\right)^{2}}{(N+1)^{2}}$ from $\left.c_{1}\right|_{\left[s_{j}, s_{j+1}\right]}$ to $\left.c_{2}\right|_{\left[s_{j}, s_{j+1}\right]}$ for $j=$ $0, \ldots, N$, where $M^{\prime}$ is a suitable universal constant. This follows from the Lipschitz 1-connectedness up to some scale of $X_{k}$. Using these homotopies, we construct a Lipschitz homotopy $\varphi_{2}^{\prime}$ from $c_{1}$ to $c_{2}$ satisfying

$$
\operatorname{Area}\left(\varphi_{2}^{\prime}\right) \leq(N+1) \cdot M^{\prime} \cdot \frac{\ell\left(c_{1}\right)^{2}}{(N+1)^{2}}=M^{\prime} \cdot \frac{\ell\left(c_{1}\right)^{2}}{N+1}
$$

We consider the Lipschitz map $\varphi_{2}$ defined on $A_{m}=\bar{D} \backslash \bigcup_{j=1}^{m} D_{j}$. By construction, we have 


$$
\sum_{j=1}^{m} \ell\left(\left.\varphi_{2}\right|_{\partial D_{j}}\right) \leq \ell\left(c_{1}\right)
$$

and each $\left.\varphi_{2}\right|_{\partial D_{j}}$ is a Jordan curve in $X$. Thus, by Proposition 5.2, there exists a Lipschitz extension $\psi_{j}: \bar{D}_{j} \rightarrow X_{k}$ of $\left.\varphi_{2}\right|_{\partial D_{j}}$ with

$$
\operatorname{Area}\left(\psi_{j}\right) \leq K \cdot \ell\left(\left.\varphi_{2}\right|_{\partial D_{j}}\right)^{2}
$$

for some constant $K$ depending only on $C$.

Finally, gluing the Lipschitz maps $\varphi_{1}, \varphi_{2}^{\prime}, \varphi_{2}$, and $\psi_{1}, \ldots, \psi_{m}$, we obtain a Lipschitz extension $\varphi: \bar{D} \rightarrow X_{k}$ of $c$ satisfying

$$
\begin{aligned}
\operatorname{Area}(\varphi) & \leq \operatorname{Area}\left(\varphi_{1}\right)+\operatorname{Area}\left(\varphi_{2}^{\prime}\right)+\operatorname{Area}\left(\varphi_{2}\right)+\sum_{j=1}^{m} \operatorname{Area}\left(\psi_{j}\right) \\
& \leq M \cdot \ell(c)^{2}+M^{\prime}(N+1)^{-1} \cdot \ell\left(c_{1}\right)^{2}+K \cdot \sum_{j=1}^{m} \ell\left(\left.\varphi_{2}\right|_{\partial D_{j}}\right)^{2} \\
& \leq\left(M+4 M^{\prime}+4 K\right) \cdot \ell(c)^{2}
\end{aligned}
$$

This proves that $X_{k}$ admits a quadratic isoperimetric inequality with isoperimetric constant at most $C^{\prime}=M+4 M^{\prime}+4 K$. This completes the proof.

Theorem 5.1 has the following consequence.

\section{COROLLARY 5.5}

Let $X$ be a complete, geodesic metric space homeomorphic to $\bar{D}, S^{2}$, or $\mathbb{R}^{2}$. Suppose that $X$ is linearly locally connected and there exists $L>0$ such that

$$
\mathscr{H}^{2}(B(x, r)) \leq L \cdot r^{2}
$$

for every $x \in X$ and $r>0$. Then $X$ admits a quadratic isoperimetric inequality.

The corollary follows directly from Theorem 5.1 together with the lemma below.

\section{LEMMA 5.6}

Let $X$ be a proper metric space homeomorphic to $\bar{D}, S^{2}$, or $\mathbb{R}^{2}$. Suppose that $X$ is linearly locally connected and there exists $L>0$ such that

$$
\mathscr{H}^{2}(B(x, r)) \leq L \cdot r^{2}
$$

for every $x \in X$ and $r>0$. Then there exists $C>0$ such that every Jordan curve $\Gamma \subset X$ bounds a Jordan domain $\Omega \subset X$ satisfying

$$
\mathscr{H}^{2}(\Omega) \leq C \cdot \ell(\Gamma)^{2} .
$$


If $X$ is homeomorphic to $S^{2}$ or $\mathbb{R}^{2}$, then the lemma holds with $C=L \lambda^{2}$, where $\lambda$ is the linear local connectedness constant of $X$. If $X$ is homeomorphic to $\bar{D}$, then the constant $C$ which we obtain in our proof depends on $L, \lambda, \mathscr{H}^{2}(X)$, and $\operatorname{diam}(\partial X)$.

\section{Proof}

We only give the proof in the case that $X$ is homeomorphic to $\bar{D}$, the argument for the other cases being similar but simpler. Let $\Omega \subset X$ be a Jordan domain, and set $r:=\ell(\partial \Omega)$. Let $\lambda \geq 1$ be the linear local connectedness constant. We distinguish two cases and first assume that $r<(2 \lambda)^{-1} \cdot \operatorname{diam}(\partial X)$. Fix a point $x \in \partial \Omega$ and observe that there exists $x^{\prime} \in \partial X$ with $d\left(x, x^{\prime}\right)>\lambda r$. Since $X$ is a disk, $\Omega$ cannot intersect the boundary circle $\partial X$, and hence $x^{\prime} \notin \Omega$. Since $\partial \Omega \subset B(x, r)$, it thus follows that $x^{\prime} \in X \backslash \bar{\Omega}$. We now show that $\Omega \subset B(x, \lambda r)$. We argue by contradiction and assume that there exists $x^{\prime \prime} \in \Omega \backslash B(x, \lambda r)$. Since $X$ is $\lambda$-linearly locally connected, there exists a continuum $E \subset X \backslash B(x, r)$ connecting $x^{\prime}$ and $x^{\prime \prime}$. However, $E$ must intersect $\partial \Omega$, which contradicts the fact that $\partial \Omega \subset B(x, r)$. Hence, $\Omega \subset B(x, \lambda r)$. Finally, we estimate

$$
\mathscr{H}^{2}(\Omega) \leq \mathscr{H}^{2}(B(x, \lambda r)) \leq L \cdot(\lambda r)^{2}=L \lambda^{2} \cdot \ell(\partial \Omega)^{2}
$$

This concludes the proof of the first case. We now assume that $r \geq(2 \lambda)^{-1} \cdot \operatorname{diam}(\partial X)$. Observe that $\mathscr{H}^{2}(X)<\infty$, and hence

$$
\mathscr{H}^{2}(\Omega) \leq \mathscr{H}^{2}(X) \leq \frac{4 \lambda^{2} \cdot \mathscr{H}^{2}(X)}{\operatorname{diam}(\partial X)^{2}} \cdot \ell(\partial \Omega)^{2} .
$$

This concludes the proof.

The following proposition will be needed in the proof of Theorem 1.4.

PROPOSITION 5.7

Let $X$ be as in Theorem 1.4, and let $c: S^{1} \rightarrow X$ be an injective Lipschitz curve. Then for every $\varepsilon>0$, there exists $u \in N^{1,2}(D, X)$ with $\operatorname{tr}(u)=c$ and such that

$$
\operatorname{Area}(u) \leq C \cdot \ell(c)^{2}+\varepsilon
$$

Proof

Let $c: S^{1} \rightarrow X$ be an injective Lipschitz curve. By Lemma 3.2, we may assume that $c$ is parameterized proportional to its arc-length.

Let $\Omega \subset X$ be the Jordan domain of smallest area and boundary $c$. Denote by $d_{\bar{\Omega}}$ the length metric on $\bar{\Omega}$. Set $Y:=(\bar{\Omega}, d)$ and $Z:=\left(\bar{\Omega}, d_{\bar{\Omega}}\right)$, where $d$ is the metric from $X$. By Lemma 2.1, the identity map $\iota: Z \rightarrow Y$ is a homeomorphism which preserves the lengths of curves and the Hausdorff 2-measure of Borel subsets. Moreover, 
$Z$ is geodesic and $\iota$ is 1 -Lipschitz. Finally, $\iota$ is locally isometric on $Z \backslash \partial Z$. Hence, every Jordan domain $U \subset Z$ satisfies

$$
\mathscr{H}_{Z}^{2}(U) \leq C \cdot \ell(\partial U)^{2} .
$$

It thus follows from Theorem 5.1 that $Z$ admits a quadratic isoperimetric inequality. In particular, $\Lambda(\partial Z, Z)$ is nonempty and, by Theorem 3.4, there exists $v \in \Lambda(\partial Z, Z)$ which minimizes the Reshetnyak energy $E_{+}^{2}$ among all maps in $\Lambda(\partial Z, Z)$. Moreover, $v$ has a unique representative which is continuous on $D$ and extends to a continuous map $v: \bar{D} \rightarrow Z$. Finally, $v$ satisfies Lusin's condition (N) (see [30, Theorem 4.4]). Theorem 1.2 shows that $v$ is a cell-like map and thus monotone. The area formula now implies that

$$
\operatorname{Area}(v)=\int_{v(D)} N(v, z) d \mathscr{H}_{Z}^{2}(z),
$$

where $N(v, z)$ denotes the number of points in the fiber $v^{-1}(z)$. Since $v$ is monotone and $\operatorname{Area}(v)<\infty$, it follows that $N(v, z)=1$ for almost every $z \in v(D)$ and hence

$$
\operatorname{Area}(v)=\mathscr{H}^{2}(Z)=\mathscr{H}_{Y}^{2}(\bar{\Omega}) \leq C \cdot \ell(c)^{2} .
$$

Let $\varepsilon>0$. By [31, Lemma 4.8], we may connect $c$ and $\operatorname{tr}(v)$ by a Sobolev annulus $w$ of area at most $\varepsilon$. Gluing $v$ and $w$ we obtain a Sobolev map $u \in N^{1,2}(D, X)$ whose trace equals $c$ and with area at most $C \cdot \ell(c)^{2}+\varepsilon$. This completes the proof.

We can finally provide the following.

\section{Proof of Theorem 1.4}

Let $c: S^{1} \rightarrow X$ be a Lipschitz curve. Fix $N \in \mathbb{N}$ sufficiently large (see below), and let $s_{0}, \ldots, s_{N} \in S^{1}$ be equidistant points. Let $\varphi: A_{m} \rightarrow X$ be a Lipschitz map of zero area as in Lemma 5.3, when applied to the curve $c$. Set $c_{1}:=\left.\varphi\right|_{S^{1}}$, and note that $\ell\left(c_{1}\right) \leq \ell(c)$.

By Theorem 5.1, the space $X$ admits a quadratic isoperimetric inequality with isoperimetric constant only depending on $C$. Arguing as in the third paragraph of the proof of Theorem 5.1, but using the isoperimetric inequality instead of Lipschitz 1connectedness, one shows the following. There exists a Sobolev homotopy $\varphi^{\prime}$ from $c$ to $c_{1}$ satisfying

$$
\operatorname{Area}\left(\varphi^{\prime}\right) \leq C^{\prime}(N+1)^{-1} \cdot \ell(c)^{2}
$$

for some constant $C^{\prime}$ depending only on $C$, whenever $N$ was chosen large enough. 
Let $\varepsilon>0$, and write $A_{m}$ as $A_{m}=\bar{D} \backslash \bigcup_{j=1}^{m} D_{j}$. By Proposition 5.7, there exists for each $j$ a map $u_{j} \in N^{1,2}\left(D_{j}, X\right)$ with $\operatorname{tr}\left(u_{j}\right)=\left.\varphi\right|_{\partial D_{j}}$ and

$$
\operatorname{Area}\left(u_{j}\right) \leq C \cdot \ell\left(\left.\varphi\right|_{\partial D_{j}}\right)^{2}+\frac{\varepsilon}{m}
$$

Gluing the maps $\varphi, \varphi^{\prime}$, and $u_{1}, \ldots, u_{m}$ yields a Sobolev map $u \in N^{1,2}(D, X)$ with $\operatorname{tr}(u)=c$ and such that

$$
\operatorname{Area}(u) \leq \operatorname{Area}\left(\varphi^{\prime}\right)+\sum_{j=1}^{m} \operatorname{Area}\left(u_{j}\right) \leq\left[C+\varepsilon+C^{\prime}(N+1)^{-1}\right] \cdot \ell(c)^{2} .
$$

Choosing $\varepsilon>0$ arbitrarily small and $N$ arbitrarily large, we see that $X$ admits a quadratic isoperimetric inequality with constant arbitrarily close to $C$. It thus follows from [7, Theorem 4.5] that $X$ actually admits a quadratic isoperimetric inequality with constant equal to $C$. This completes the proof.

Combining Theorem 1.4, Theorem 3.4, and Theorem 1.2, we obtain the following almost parameterization result.

\section{THEOREM 5.8}

Let $X$ be as in Theorem 1.4 and such that $X$ is homeomorphic to $\bar{D}$ and $\ell(\partial X)<\infty$. Then $\Lambda(\partial X, X)$ contains an element $u$ of minimal energy $E_{+}^{2}(u)$. Every such $u$ is infinitesimally isotropic and has a continuous representative which is a uniform limit of homeomorphisms from $\bar{D}$ to $X$.

The following example (see, e.g., [31, Example 11.3]) illustrates that spaces as in the theorem need not be Ahlfors 2-regular.

\section{Example 5.9}

Let $T \subset D$ be a compact ball. Denote by $X$ the metric space obtained from $\bar{D}$ by identifying points in $T$, equipped with the quotient metric. Then $X$ is a geodesic metric space which is homeomorphic to $\bar{D}$ and satisfies (1).

Clearly, the space $X$ in the example is not Ahlfors 2-regular. Moreover, $X$ is not reciprocal as defined in [37] and hence does not admit a quasiconformal parameterization in the sense of [37].

\section{Proofs of parameterization results}

The following result makes the statements in Theorem 1.1 more precise and slightly more general. 


\section{THEOREM 6.1}

Let $X$ be an Ahlfors 2-regular, geodesic metric space homeomorphic to a 2dimensional manifold. Let $\Omega \subset X$ be a Jordan domain with $\ell(\partial \Omega)<\infty$ and such that $\bar{\Omega}$ is linearly locally connected. Then there exists $u \in \Lambda(\partial \Omega, \bar{\Omega})$ which is continuous on $\bar{D}$ and satisfies

$$
E_{+}^{2}(u)=\inf \left\{E_{+}^{2}(v): v \in \Lambda(\partial \Omega, \bar{\Omega})\right\}
$$

Any such map is a quasisymmetric homeomorphism from $\bar{D}$ to $\bar{\Omega}$ and is uniquely determined up to a conformal diffeomorphism of $\bar{D}$.

By a manifold we mean a topological manifold with or without boundary.

\section{Proof}

Denote by $Y$ the set $\bar{\Omega}$ equipped with the metric from $X$ and by $Z$ the same set $\bar{\Omega}$ equipped with the length metric. By Lemma 2.1, the identity map $\iota: Z \rightarrow Y$ is a homeomorphism which preserves the lengths of curves and the Hausdorff 2-measure of Borel subsets. Moreover, $Z$ is geodesic and $\iota$ is 1-Lipschitz.

By Lemma 5.6, there exists $C>0$ such that every Jordan domain $U \subset Y$ satisfies

$$
\mathscr{H}^{2}(U) \leq C \cdot \ell(\partial U)^{2} .
$$

Hence, the same is true for Jordan domains in the space $Z$. Thus, Theorem 5.1 shows that $Z$ admits a quadratic isoperimetric inequality. It follows that also $Y$ admits a quadratic isoperimetric inequality. By Theorem 3.4, there exists $u \in \Lambda(\partial Y, Y)$ which minimizes the Reshetnyak energy $E_{+}^{2}$ among all maps in $\Lambda(\partial Y, Y)$. Moreover, any such $u$ is infinitesimally isotropic and has a unique representative which is continuous on $D$ and extends to a continuous map $u: \bar{D} \rightarrow Y$.

We will now show that any $u$ with the properties above is a quasisymmetric homeomorphism. For this, consider the map $v:=\iota^{-1} \circ u$, which is continuous, in $N^{1,2}\left(D, Z\right.$ ), and satisfies ap md $v_{z}=$ apmd $u_{z}$ for almost every $z \in D$ (see [31, Corollary 3.2]). In particular, $v \in \Lambda(\partial Z, Z)$ and $E_{+}^{2}(v)=E_{+}^{2}(u)$. It is now clear that $v$ is an energy minimizer in $\Lambda(\partial Z, Z)$ since for any $w \in \Lambda(\partial Z, Z)$ we have

$$
E_{+}^{2}(v)=E_{+}^{2}(u) \leq E_{+}^{2}(\iota \circ w) \leq E_{+}^{2}(w)
$$

Thus, Theorem 1.2 shows that $v$ is a uniform limit of homeomorphisms from $\bar{D}$ to $Z$ and thus monotone by Proposition 2.9. Consequently, the map $u$ is monotone too. Since $u$ is infinitesimally isotropic and thus infinitesimally quasiconformal, Theorem 3.6 shows that $u$ is a homeomorphism from $\bar{D}$ to $Y$. Proposition 3.5 and Theorem 2.5 imply that $u$ is quasisymmetric.

We are left to show that any map as above is unique up to composition with a conformal diffeomorphism of $D$. Thus, let $u$ and $v$ be energy minimizers in $\Lambda(\partial Y, Y)$ 
which are continuous on $\bar{D}$. They are thus quasisymmetric homeomorphisms from $\bar{D}$ to $Y$ by the argument above. We will show that the map $\varphi: \bar{D} \rightarrow \bar{D}$ given by $\varphi:=v^{-1} \circ u$ is a conformal diffeomorphism of $D$. First notice that, as the composition of two quasisymmetric homeomorphisms, the map $\varphi$ is itself quasisymmetric and, in particular, $\varphi$ and $\varphi^{-1}$ satisfy Lusin's condition (N) (see [45, Theorem 33.2]). It thus follows from the approximate metric differentiability of $u$ and $v$ that

$$
\text { ap md } u_{z}=\text { ap } \operatorname{md} v_{\varphi(z)} \circ d_{z} \varphi
$$

for almost every $z \in D$ (see [29, Proposition 4.3]). Here, $d_{z} \varphi$ denotes the classical differential of $\varphi$, which exists for almost every $z \in D$ (see [45, Corollary 32.2]). By the area formula and [16, Corollary 8.15], the approximate metric derivatives ap $m d u_{z}$ and ap md $v_{z}$ are nondegenerate at almost every $z$.

By Theorem 3.4, the maps $u$ and $v$ are infinitesimally isotropic, so for almost every $z$ the unique ellipses of largest area (John's ellipses) contained in the unit balls with respect to the norms ap $m d u_{z}$ and ap $m d v_{\varphi(z)}$ are given by Euclidean disks. Since the map

$$
d_{z} \varphi:\left(\mathbb{R}^{2}, \text { ap } \operatorname{md} u_{z}\right) \rightarrow\left(\mathbb{R}^{2}, \text { ap } \operatorname{md} v_{\varphi(z)}\right)
$$

is an isometry, it must map John's ellipses to John's ellipses. This shows that $\varphi$ is 1-quasiconformal and hence a conformal diffeomorphism. This proves that $u$ and $v$ indeed agree up to a conformal diffeomorphism.

We now prove an analogue of Theorem 6.1 for spaces homeomorphic to $S^{2}$. Given a complete metric space $X$, denote by $N^{1,2}\left(S^{2}, X\right)$ the Newton-Sobolev space defined as in Section 3 with $\Omega$ replaced by $S^{2}$. The energy $E_{+}^{2}(u)$ of an element $u \in N^{1,2}\left(S^{2}, X\right)$ is defined analogously. Let $\Lambda(X)$ be the family of maps $u \in N^{1,2}\left(S^{2}, X\right)$ such that $u$ is a uniform limit of homeomorphisms from $S^{2}$ to $X$.

\section{THEOREM 6.2}

Let $X$ be an Ahlfors 2-regular, linearly locally connected metric space homeomorphic to $S^{2}$. Then $\Lambda(X)$ is not empty and contains an element $u$ which satisfies

$$
E_{+}^{2}(u)=\inf \left\{E_{+}^{2}(v): v \in \Lambda(X)\right\} .
$$

Any such map $u$ is a quasisymmetric homeomorphism from $S^{2}$ to $X$ and is uniquely determined up to a conformal diffeomorphism of $S^{2}$.

Since quasisymmetric homeomorphisms preserve the linear local connectedness, our theorem, in particular, implies the Bonk-Kleiner theorem (see [4]), restated in the 
corollary below. Actually, the corollary will already follow from the first step of the proof of our theorem (see Remark 6.5).

\section{COROLLARY 6.3}

Let $X$ be an Ahlfors 2-regular metric space homeomorphic to $S^{2}$. Then $X$ is quasisymmetric to $S^{2}$ if and only if $X$ is linearly locally connected.

We turn to the proof of Theorem 6.2 and first show the following.

PROPOSITION 6.4

If $X$ is as in Theorem 6.2, then $\Lambda(X)$ is not empty.

\section{Proof}

By [41, Theorem B.6], the space $X$ is quasi-convex. Thus, after changing the metric on $X$ in a bi-Lipschitz way, we may assume that $X$ is geodesic. Starting with a Jordan curve in $X$ and arguing exactly as in the proof of Lemma 4.2 we find a Jordan curve $\Gamma \subset X$ which is moreover a bi-Lipschitz curve. Let $\Omega_{1}, \Omega_{2} \subset X$ be the two Jordan domains enclosed by $\Gamma$. We claim that $\bar{\Omega}_{1}$ and $\bar{\Omega}_{2}$ are linearly locally connected. Indeed, for $i=1,2$ one constructs continua in $\bar{\Omega}_{i}$ satisfying the linear local connectedness condition by using the continua in $X$ given by the linear local connectedness and replacing the part outside $\bar{\Omega}_{i}$ by a part of the bi-Lipschitz curve $\partial \Omega_{i}$. By Theorem 6.1, there exist quasisymmetric homeomorphisms $u_{k}: \bar{D} \rightarrow \bar{\Omega}_{k}$ for $k=1,2$. The map given by $\varphi:=\left.u_{2}^{-1} \circ u_{1}\right|_{S^{1}}$ is a quasisymmetric homeomorphism of $S^{1}$ to itself and hence extends to a quasisymmetric homeomorphism $\bar{\varphi}$ of $\bar{D}$ by [3]. Consequently, the quasisymmetric homeomorphism $\bar{u}_{2}: \bar{D} \rightarrow \bar{\Omega}_{2}$ defined by $\bar{u}_{2}:=u_{2} \circ \bar{\varphi}$ agrees with $u_{1}$ on the boundary $S^{1}$. Now, identify $S^{2}$ in a bi-Lipschitz way with the space obtained by gluing two copies of $\bar{D}$ along their common boundary $S^{1}$. Let $\psi: S^{2} \rightarrow X$ be the homeomorphism which coincides with $\bar{u}_{2}$ on one copy of $\bar{D}$ and with $u_{1}$ on the other copy. Since $u_{1}$ and $\bar{u}_{2}$ are Sobolev maps, it follows from the general gluing theorem for Sobolev maps (see [22, Theorem 12.1.3]) that $\psi$ is in $N^{1,2}\left(S^{2}, X\right)$ and hence $\Lambda(X)$ is not empty. This completes the proof.

\section{Remark 6.5}

Notice that the map $\psi$ constructed in the proof of the proposition satisfies the hypotheses of the quasisymmetric gluing theorem (see [1, Theorem 3.1]) and thus $\psi$ is actually a quasisymmetric homeomorphism. This already establishes Corollary 6.3.

Now, we identify $S^{2}$ with the Riemann sphere $\hat{\mathbb{C}}$ and note that precompositions with conformal maps in domains of $\hat{\mathbb{C}}$ preserve Sobolev maps and the Reshetnyak 
energy (see [29]). This allows us to reduce all local questions and statements about elements in $N^{1,2}\left(S^{2}, X\right)$ to the case of Sobolev maps on bounded domains in $\mathbb{C}=$ $\mathbb{R}^{2}$. In particular, the Reshetnyak energy is lower semicontinuous for energy bounded sequences in $N^{1,2}\left(S^{2}, X\right)$ (see [17], [29]), and any map $u \in N^{1,2}\left(S^{2}, X\right)$ has an approximate metric derivative almost everywhere. As in the case of disks, we have the following.

\section{THEOREM 6.6}

Let $u \in N^{1,2}\left(S^{2}, X\right)$ be such that $E_{+}^{2}(u \circ \phi) \geq E_{+}^{2}(u)$ for all bi-Lipschitz homeomorphisms $\phi: S^{2} \rightarrow S^{2}$. Then u is infinitesimally isotropic.

Indeed, the proof of [29, Theorem 1.2], repeated and reformulated in [30, Lemmas 3.2, 4.1], applies to the present situation without change, since the "critical" bi-Lipschitz homeomorphism $\phi$ of $\bar{D}$ constructed in [29] is fractional linear outside a small ball and hence extends to a bi-Lipschitz homeomorphism of $\hat{\mathbb{C}}$ which is conformal outside a small ball.

\section{Proof of Theorem 6.2}

By Proposition 6.4, the family $\Lambda(X)$ is not empty. The existence of an energy minimizer in $\Lambda(X)$ is now proved as in the classical case when $X$ is smooth (see [18, Section 3.1]). Indeed, fix distinct points $p_{1}, p_{2}, p_{3} \in S^{2}$ and distinct points $q_{1}, q_{2}, q_{3} \in$ $X$. Let $\left(u_{n}\right)$ be an energy minimizing sequence in $\Lambda(X)$. After possibly composing with conformal diffeomorphisms of $S^{2}$ we may assume that each $u_{n}$ satisfies the 3 point condition $u_{n}\left(p_{i}\right)=q_{i}$ for $i=1,2,3$. Applying the Courant-Lebesgue lemma, we deduce as in [18, Section 3.1] that the family $\left(u_{n}\right)$ is equicontinuous. Thus, after possibly passing to a subsequence, the sequence $\left(u_{n}\right)$ converges uniformly to a map $u: S^{2} \rightarrow X$. Then $u$ is in $N^{1,2}\left(S^{2}, X\right)$ and is a uniform limit of homeomorphisms from $S^{2}$ to $X$, and hence $u \in \Lambda(X)$. By the lower semicontinuity of $E_{+}^{2}$ we thus see that $u$ is an energy minimizer in $\Lambda(X)$. This proves the existence of an energy minimizer in $\Lambda(X)$.

Let $u$ be any energy minimizer in $\Lambda(X)$. By Theorem 6.6, the map $u$ is infinitesimally isotropic. Since $u$ is monotone, it follows as in the proof of Theorem 6.1 that $u$ is a quasisymmetric homeomorphism and that $u$ is unique up to precomposition with a conformal diffeomorphism.

\section{Appendix}

The purpose of this section is to establish the following. 


\section{Proof of Theorem 2.5}

Since quasisymmetric maps preserve linear local connectedness, one direction is clear. In order to prove the other direction, suppose that $X$ is linearly locally connected with some constant $\lambda \geq 1$. The linear local connectedness and the quadratic upper bound on the Hausdorff measure together imply that $X$ is Ahlfors 2-regular and thus doubling. This follows similarly to [37, p. 1369]. For $z \in \bar{D}$ and $r>0$, define

$$
L(z, r):=\max \left\{d\left(u(z), u\left(z^{\prime}\right)\right): z^{\prime} \in \bar{D} \cap \bar{B}(z, 2 r)\right\},
$$

where $\bar{B}(z, 2 r)$ denotes the closed ball. Since $u$ is uniformly continuous there exists $r>0$ such that

$$
L(z, r)<\frac{\operatorname{diam} X}{4}
$$

for all $z \in \bar{D}$. By [14, Theorem 10.19] and [44, Theorem 2.23], it suffices to show that for every $z \in \bar{D}$ the restriction of $u$ to $\bar{D} \cap B(z, r)$ is a weak quasisymmetry. See [14, Section 10] for the definition of a weak quasisymmetry.

Fix $z \in \bar{D}$, and let $w, a, b \in \bar{D} \cap B(z, r)$ be such that

$$
|w-a| \leq|w-b| \text {. }
$$

Set $s:=d(u(w), u(a))$, and let $M>0$ be such that

$$
s>M \cdot d(u(w), u(b)) .
$$

It suffices to show that $M$ must be bounded from above by a constant depending only on $Q, L$, and $\lambda$. If $M \leq 4 \lambda^{2}$, then nothing needs to be proved, so we may assume that $M>4 \lambda^{2}$.

We claim that there exists $z^{\prime} \in \bar{D} \backslash \bar{B}(z, 2 r)$ with $u\left(z^{\prime}\right) \notin \bar{B}(u(w), s / 2)$. Indeed, due to (5), there exists $y \in X$ with $d(y, u(z))>2 L(z, r)$ and thus $z^{\prime}:=u^{-1}(y)$ satisfies $z^{\prime} \notin \bar{B}(z, 2 r)$. Consequently,

$$
2 L(z, r)<d(y, u(z)) \leq d(y, u(w))+d(u(w), u(z)) \leq d(y, u(w))+L(z, r),
$$

from which it follows together with

$$
s \leq d(u(w), u(z))+d(u(z), u(a)) \leq 2 L(z, r)
$$

that

$$
d(y, u(w))>L(z, r) \geq \frac{s}{2}
$$

This proves the claim. 
Since $u(b) \in B(u(w), s / M)$ and $u(a), u\left(z^{\prime}\right) \notin B(u(w), s / 2)$, it follows from the linear local connectedness of $X$ that there exists a continuum

$$
E^{\prime} \subset B(u(w), \lambda s / M)
$$

connecting $u(w)$ and $u(b)$ and there exists a continuum

$$
F^{\prime} \subset X \backslash B(u(w), s / 2 \lambda)
$$

connecting $u(a)$ and $u\left(z^{\prime}\right)$. Let $\Gamma\left(E^{\prime}, F^{\prime} ; X\right)$ be the family of curves joining $E^{\prime}$ to $F^{\prime}$ in $X$. Since $\Gamma\left(E^{\prime}, F^{\prime} ; X\right)$ is a subset of the family of curves joining $\bar{B}(u(w), \lambda s / M)$ to $X \backslash B(u(w), s / 2 \lambda)$ in $X$, it follows from [14, Lemma 7.18] that

$$
\bmod \left(\Gamma\left(E^{\prime}, F^{\prime} ; X\right)\right) \leq L^{\prime} \cdot\left(\log \frac{M}{2 \lambda^{2}}\right)^{-1}
$$

where $L^{\prime}$ is a constant depending on $L$.

Set $E:=u^{-1}\left(E^{\prime}\right)$ and $F:=u^{-1}\left(F^{\prime}\right)$. Then $\Gamma\left(E^{\prime}, F^{\prime} ; X\right)=u \circ \Gamma(E, F ; \bar{D})$, and hence

$$
\bmod (\Gamma(E, F ; \bar{D})) \leq Q \cdot \bmod \left(\Gamma\left(E^{\prime}, F^{\prime} ; X\right)\right)
$$

We clearly have

$$
\operatorname{dist}(E, F) \leq 2 \cdot \min \{\operatorname{diam} E, \operatorname{diam} F\}
$$

since $\operatorname{dist}(E, F) \leq|w-a|$ and

$$
\min \{\operatorname{diam} E, \operatorname{diam} F\} \geq \min \left\{|w-b|,\left|a-z^{\prime}\right|\right\} \geq \frac{1}{2} \cdot|w-a| .
$$

It thus follows that

$$
\bmod (\Gamma(E, F ; \bar{D})) \geq \phi(2)>0,
$$

where $\phi$ is a Loewner function for $\bar{D}$. For a definition of the Loewner function and the Loewner property of $\bar{D}$ see, for example, [14]. Combining inequalities (6), (7), and (8), we see that

$$
M \leq 2 \lambda^{2} \cdot \exp \left(\frac{Q L^{\prime}}{\phi(2)}\right) .
$$

This shows that the restriction of $u$ to $\bar{D} \cap B(z, r)$ is weakly $H$-quasisymmetric for some $H$ only depending on $\lambda, Q, L$. 
Acknowledgments. The authors wish to thank Katrin Fässler, Anton Petrunin, Pekka Koskela, Kai Rajala, and Stephan Stadler for discussions and several anonymous referees for the careful reading of our manuscript and helpful comments. Parts of this paper were written while the second author was visiting the Scuola Normale Superiore di Pisa. He wishes to thank the institute for the hospitality and the inspiring atmosphere he enjoyed during his visit.

The first author was partially supported by Deutsche Forschungsgemeinschaft grant SPP 2026. The second author was partially supported by Swiss National Science Foundation grants 153599 and 165848.

\section{References}

[1] V. V. ASEEV, D. G. KUZIN, and A. V. TETENOV, Angles between sets and the gluing of quasisymmetric mappings in metric spaces, Izv. Vyssh. Uchebn. Zaved. Mat. 2005, no. 10, 3-13; English translation in Russian Math. (Iz. VUZ) 49 (2005), no. 10, 1-10. MR 2229038. (790)

[2] Y. BENYAMINI and J. LINDENSTRAUSS, Geometric Nonlinear Functional Analysis, Vol. 1, Amer. Math. Soc. Colloq. Publ. 48, Amer. Math. Soc., Providence, 2000. MR 1727673. (775)

[3] A. BEURLING and L. AHLFORS, The boundary correspondence under quasiconformal mappings, Acta Math. 96 (1956), 125-142. MR 0086869.

DOI 10.1007/BF02392360. (790)

[4] M. BONK and B. KLEINER, Quasisymmetric parametrizations of two-dimensional metric spheres, Invent. Math. 150 (2002), no. 1, 127-183. MR 1930885. DOI 10.1007/s00222-002-0233-z. (761, 766, 789)

[5] Conformal dimension and Gromov hyperbolic groups with 2-sphere boundary, Geom. Topol. 9 (2005), 219-246. MR 2116315. DOI 10.2140/gt.2005.9.219. (761)

[6] D. BURAGO, Y. BURAGO, and S. IVANOV, A Course in Metric Geometry, Grad. Stud. Math., 33, Amer. Math. Soc., Providence, 2001. MR 1835418. DOI 10.1090/gsm/033. (766)

[7] P. CREUTZ, Plateau's problem for singular curves, preprint, arXiv:1904.12567 [math.DG]. (787)

[8] R. J. DAVERMAN, Decompositions of Manifolds, AMS Chelsea Publishing, Providence, 2007. MR 2341468. DOI 10.1090/chel/362. (768, 769)

[9] R. D. EDWARDS, "The topology of manifolds and cell-like maps" in Proceedings of the International Congress of Mathematicians (Helsinki, 1978), Acad. Sci. Fennica, Helsinki, 1980, 111-127. MR 0562601. (768, 769)

[10] L. C. EVANS and R. F. GARIEPY, Measure Theory and Fine Properties of Functions, Stud. Adv. Math., CRC Press, Boca Raton, FL, 1992. MR 1158660. (770)

[11] E. E. FLOYD, Some characterizations of interior maps, Ann. of Math. (2) 51 (1950), 571-575. MR 0035007. DOI 10.2307/1969369. (778) 
[12] K. P. HART, J.-I. NAGATA, and J. E. VAUGHAN, eds., Encyclopedia of General Topology, Elsevier, Amsterdam, 2004. MR 2049453. (768, 779)

[13] L. I. HEDBERG and T. KILPELÄINEN, On the stability of Sobolev spaces with zero boundary values, Math. Scand. 85 (1999), no. 2, 245-258. MR 1724237. DOI 10.7146/math.scand.a-18274. (775)

[14] J. HEINONEN, Lectures on Analysis on Metric Spaces, Springer, New York, 2001. MR 1800917. DOI 10.1007/978-1-4613-0131-8. (766, 774, 792, 793)

[15] J. HEINONEN and P. KOSKELA, Quasiconformal maps in metric spaces with controlled geometry, Acta Math. 181 (1998), no. 1, 1-61. MR 1654771. DOI 10.1007/BF02392747. (763, 766, 768)

[16] J. HEINONEN, P. KOSKELA, N. SHANMUGALINGAM, and J. TYSON, Sobolev classes of Banach space-valued functions and quasiconformal mappings, J. Anal. Math. 85 (2001), 87-139. MR 1869604. DOI 10.1007/BF02788076. (770, 789)

[17] - Sobolev Spaces on Metric Measure Spaces, New Math. Monogr. 27, Cambridge Univ. Press, Cambridge, 2015. MR 3363168. DOI 10.1017/CBO9781316135914. (762, 766, 767, 770, 771, 773, 791)

[18] J. JOST, Two-Dimensional Geometric Variational Problems, Pure Appl. Math. (N.Y.), Wiley, Chichester, 1991. MR 1100926. (761, 791)

[19] J. JOST and R. SCHOEN, On the existence of harmonic diffeomorphisms, Invent. Math. 66 (1982), no. 2, 353-359. MR 0656629. DOI 10.1007/BF01389400. (762)

[20] M. B. KARMANOVA, Area and co-area formulas for mappings of the Sobolev classes with values in a metric space (in Russian), Sibirsk. Mat. Zh. 48 (2007), no. 4, 778-788; English translation in Siberian Math. J. 48 (2007), no. 4, 621-628. MR 2355373. DOI 10.1007/s11202-007-0064-7. (770, 771, 773)

[21] B. KIRCHHEIM, Rectifiable metric spaces: Local structure and regularity of the Hausdorff measure, Proc. Amer. Math. Soc. 121 (1994), no. 1, 113-123. MR 1189747. DOI 10.2307/2160371. (765, 771, 773)

[22] N. J. KOREVAAR and R. M. SCHOEN, Sobolev spaces and harmonic maps for metric space targets, Comm. Anal. Geom. 1 (1993), nos. 3-4, 561-659. MR 1266480. DOI 10.4310/CAG.1993.v1.n4.a4. (765, 771, 772, 790)

[23] E. KUWERT, Harmonic maps between flat surfaces with conical singularities, Math. Z. 221 (1996), no. 3, 421-436. MR 1381590. DOI 10.1007/PL00004251. (762)

[24] R. C. LACHER, Cell-like mappings, I, Pacific J. Math. 30 (1969), no. 3, 717-731. MR 0251714. (777)

[25] U. LANG and T. SCHLICHENMAIER, Nagata dimension, quasisymmetric embeddings, and Lipschitz extensions, Int. Math. Res. Not. 2005, no. 58, 3625-3655. MR 2200122. DOI 10.1155/IMRN.2005.3625. (764)

[26] A. LOHVANSUU, K. RAJALA, and M. RASIMUS, Quasispheres and metric doubling measures, Proc. Amer. Math. Soc. 146 (2018), no. 7, 2973-2984. MR 3787358. DOI 10.1090/proc/13971. (761)

[27] J. LUUKKAINEN and J. VÄISÄLÄ, Elements of Lipschitz topology, Ann. Acad. Sci. Fenn. Ser. A I Math. 3 (1977), no. 1, 85-122. MR 0515647. DOI 10.5186/aasfm.1977.0315. (780) 
[28] A. LYTCHAK and S. WENGER, Regularity of harmonic discs in spaces with quadratic isoperimetric inequality, Calc. Var. Partial Differential Equations 55 (2016), no. 4, art. 98. MR 3528439. DOI 10.1007/s00526-016-1044-1. (770, 771, 772)

[29] - Area minimizing discs in metric spaces, Arch. Ration. Mech. Anal. 223 (2017), no. 3, 1123-1182. MR 3594354. DOI 10.1007/s00205-016-1054-3. $(763,770,771,772,773,789,791)$

[30] - Energy and area minimizers in metric spaces, Adv. Calc. Var. 10 (2017), no. 4, 407-421. MR 3707085. DOI 10.1515/acv-2015-0027. (763, 770, 771, $772,775,786,791)$

[31] - Intrinsic structure of minimal discs in metric spaces, Geom. Topol. 22 (2018), no. 1, 591-644. MR 3720351. DOI 10.2140/gt.2018.22.591. (763, 775, 777, 786, 787, 788)

[32] - Isoperimetric characterization of upper curvature bounds, Acta Math. 221 (2018), no. 1, 159-202. MR 3877021. DOI 10.4310/ACTA.2018.v221.n1.a5. (764)

[33] A. LYTCHAK, S. WENGER, and R. YOUNG, Dehn functions and Hölder extensions in asymptotic cones, J. Reine Angew. Math, published online 20 January 2019. DOI 10.1515/crelle-2018-0041. (764, 771, 780, 782, 783)

[34] S. MERENKOV and K. WILDRICK, Quasisymmetric Koebe uniformization, Rev. Mat. Iberoam. 29 (2013), no. 3, 859-909. MR 3090140. DOI 10.4171/RMI/743. (761)

[35] C. MESE, The structure of singular spaces of dimension 2, Manuscripta Math. 100 (1999), no. 3, 375-389. MR 1726227. DOI 10.1007/s002290050207. (762)

[36] - A variational construction of the Teichmüller map, Calc. Var. Partial Differential Equations 21 (2004), no. 1, 15-46. MR 2078745. DOI 10.1007/s00526-003-0243-8. (762)

[37] K. RAJALA, Uniformization of two-dimensional metric surfaces, Invent. Math. 207 (2017), no. 3, 1301-1375. MR 3608292. DOI 10.1007/s00222-016-0686-0. $(761,764,787,792)$

[38] E. REICH and K. STREBEL, On the Gerstenhaber-Rauch principle, Israel J. Math. 57 (1987), no. 1, 89-100. MR 0882248. DOI 10.1007/BF02769462. (762)

[39] Y. G. RESHETNYAK, Sobolev classes of functions with values in a metric space, II, Sibirsk. Mat. Zh. 45 (2004), no. 4, 855-870. MR 2091651. DOI 10.1023/B:SIMJ.0000035834.03736.b6. (771)

[40] R. SCHOEN and S. T. YAU, On univalent harmonic maps between surfaces, Invent. Math. 44 (1978), no. 3, 265-278. MR 0478219. DOI 10.1007/BF01403164. (762)

[41] S. SEMMES, Finding curves on general spaces through quantitative topology, with applications to Sobolev and Poincaré inequalities, Selecta Math. (N.S.) 2 (1996), no. 2, 155-295. MR 1414889. DOI 10.1007/BF01587936. (790)

[42] N. SHANMUGALINGAM, Newtonian spaces: An extension of Sobolev spaces to metric measure spaces, Rev. Mat. Iberoamericana 16 (2000), no. 2, 243-279. MR 1809341. DOI 10.4171/RMI/275. (770) 
[43] P. TUKIA, The planar Schönflies theorem for Lipschitz maps, Ann. Acad. Sci. Fenn. Ser. A I Math. 5 (1980), no. 1, 49-72. MR 0595177. DOI 10.5186/aasfm.1980.0529. (781)

[44] P. TUKIA and J. VÄISÄLÄ, Quasisymmetric embeddings of metric spaces, Ann. Acad. Sci. Fenn. Ser. A I Math. 5 (1980), no. 1, 97-114. MR 0595180. DOI 10.5186/aasfm.1980.0531. (766, 792)

[45] J. VÄISÄLÄ, Lectures on n-Dimensional Quasiconformal Mappings, Lecture Notes in Math. 229, Springer, New York, 1971. MR 0454009. (789)

[46] S. WENGER, Gromov hyperbolic spaces and the sharp isoperimetric constant, Invent. Math. 171 (2008), no. 1, 227-255. MR 2358060.

DOI 10.1007/s00222-007-0084-8. (782)

[47] K. WILDRICK, Quasisymmetric parametrizations of two-dimensional metric planes, Proc. Lond. Math. Soc. (3) 97 (2008), no. 3, 783-812. MR 2448247. DOI 10.1112/plms/pdn023. (761)

[48] - Quasisymmetric structures on surfaces, Trans. Amer. Math. Soc. 362 (2010), no. 2, 623-659. MR 2551500. DOI 10.1090/S0002-9947-09-04861-2. (761)

[49] M. WILLIAMS, Geometric and analytic quasiconformality in metric measure spaces, Proc. Amer. Math. Soc. 140 (2012), no. 4, 1251-1266. MR 2869110. DOI 10.1090/S0002-9939-2011-11035-9. (772)

[50] J. W. T. YOUNGS, Homeomorphic approximations to monotone mappings, Duke Math. J. 15 (1948), no. 1, 87-94. MR 0024623. DOI 10.1215/S0012-7094-48-01511-7. (769)

[51] - The representation problem for Fréchet surfaces, Mem. Amer. Math. Soc. (1951), no. 8. MR 0043881. (768)

Lytchak

Mathematisches Institut, Universität Köln, Köln, Germany; alytchak@math.uni-koeln.de

Wenger

Department of Mathematics, University of Fribourg, Fribourg, Switzerland;

stefan.wenger@unifr.ch 
